\title{
Investigating the Molecular Mechanism of Xijiao Dihuang Decoction for the Treatment of SLE Based on Network Pharmacology and Molecular Docking Analysis
}

\author{
Fangzhi Wei, Yitian Song, Aiming Gong ${ }^{\mathbb{D}}$, Chengdan Pan, Yanping Zhuang, Xuan Zhang, \\ and Minyu Zeng
}

Department of Traditional Chinese Medicine, Hainan Medical University, Haikou 571199, China

Correspondence should be addressed to Aiming Gong; 422789075@qq.com

Received 9 September 2021; Revised 1 November 2021; Accepted 27 December 2021; Published 20 January 2022

Academic Editor: Ali Nokhodchi

Copyright $\odot 2022$ Fangzhi Wei et al. This is an open access article distributed under the Creative Commons Attribution License, which permits unrestricted use, distribution, and reproduction in any medium, provided the original work is properly cited.

\begin{abstract}
Objective. To elucidate the main mechanism of Xijiao Dihuang decoction (XJDHT) for the treatment of systemic lupus erythematosus (SLE). Methods. TCMSP, BATMAN-TCM, ETCM, and TCMID databases and literature search were used to screen the potential active compounds of XJDHT, and TCMSP and SwissProt databases were searched to predict the targets of the compounds. The targets of SLE were obtained from Genegards, OMIM, and DisGeNET databases, and Venn online platform was used to obtain the intersection targets of XJDHT and SLE. Afterwards, the PPI network was constructed by using the STRING database, and the core targets were identified by network topology analysis. GO and KEGG enrichment analyses were performed through $\mathrm{R}$ software, and molecular docking of the top three core targets and their corresponding compounds were accomplished by Autodock Vina and Pymol softwares. Results. There were 30 potential active ingredients, 289 potential targets, and 129 intersection targets screened from the above databases. Network topology analysis identified 23 core targets, such as AKT1, TNF, IL6, IL1B, and INS. GO enrichment analysis obtained 2555 terms and mainly clustering on the react to lipopolysaccharide, membrane raft, and ubiquitin-like protein ligase binding. KEGG enrichment analysis obtained 187 signaling pathways, mainly concentrating on the lipid and atherosclerosis, AGE-RAGE signaling pathway in diabetic complications, fluid shear stress, and atherosclerosis. Molecular docking verified that the active compounds of XJDHT have the strong binding activity to the core targets. Conclusion. This study preliminarily uncovers the mechanism of XJDHT acting on SLE through a "multicompound, multitarget, and multipathway" manner. XJDHT may achieve the treatment of SLE by inhibiting the proinflammatory factors, inflammatory signal cvtokines, proliferation, injury, and apoptosis processes. In summary, the present study would provide a promising theoretical basis for further clinical and experimental studies.
\end{abstract}

\section{Introduction}

SLE is a chronic autoimmune disease that involves multiple organs and tissues [1]. The incidence and prevalence of SLE are different around the world. The incidence in China is about $1 / 1000$. In recent years, the prevalence of SLE has shown an upward trend [2]. The etiology and pathogenesis of SLE are not yet clear, which may be related to multiple factors such as genetic factors, environmental factors, and estrogen levels [3-5]. The treatment of SLE is still a difficult problem in the medical field, and modern medicine mostly uses glucocorticoids and immunosuppressive drugs [6], which may produce some side effects [7]. Traditional Chinese medicine (TCM) has rich experience in treating SLE with a wide variety of therapies. TCM not only improves the symptoms and the quality of survival of SLE patients but also reduces the side effects of glucocorticoid drugs and is cost-effective, thus it is a common treatment for SLE in Chinese clinics [8-10].

Xijiao Dihuang decoction (XJDHT) is from Wai Tai Mi Yao, compiled by Wang Tao in the Tang Dynasty. The formula consists of Bubali Cornu (Shuiniujiao, SNJ, 30 g), Paeoniae Radix Rubra (Chishao, CS, 10g), Dried Rehmanniae Radix (Shengdihuang, SDH, 20 g), and Moutan Cortex 


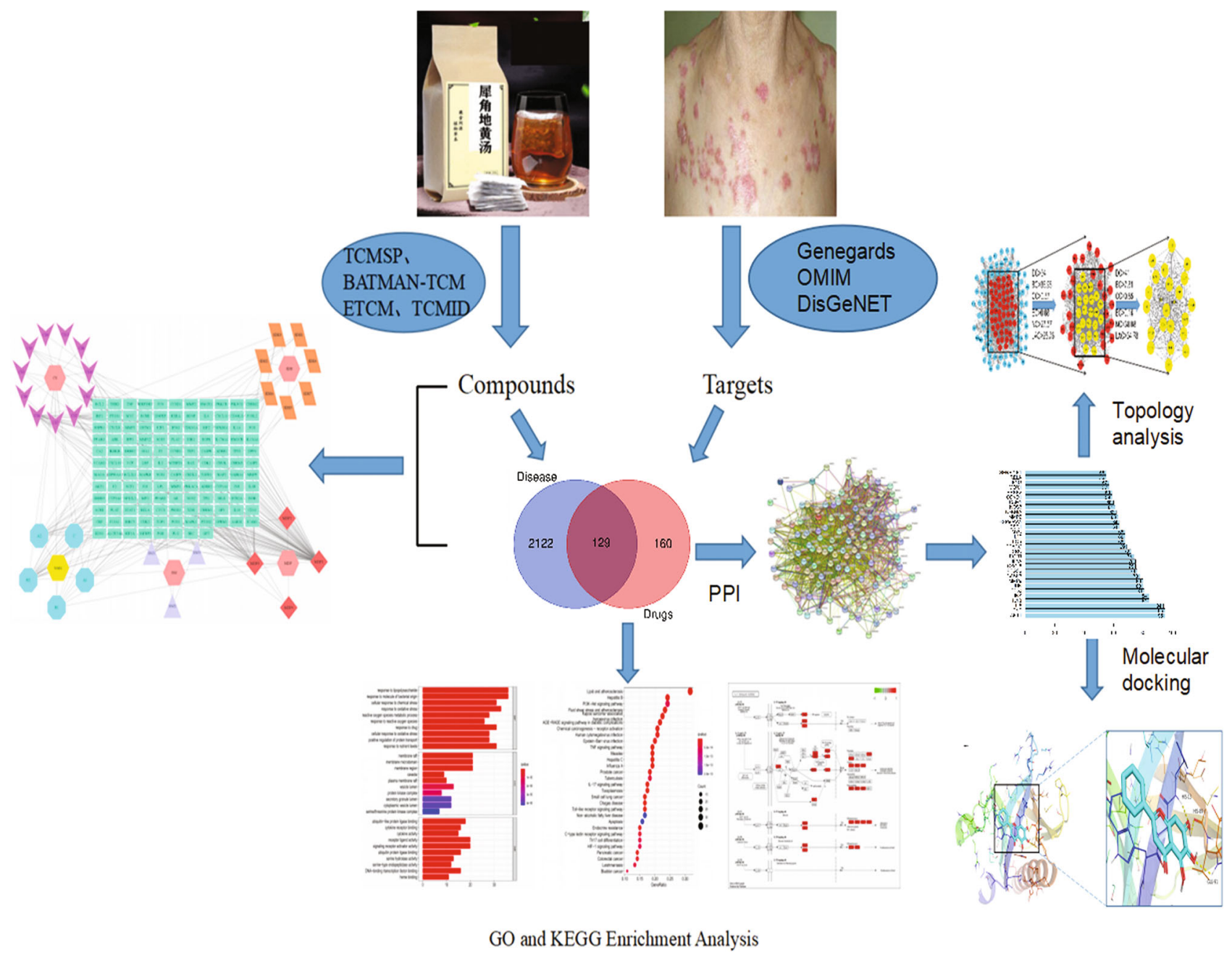

FIgURE 1: The flowchart of the analysis procedures of the study.

(Mudanpi, MDP, $15 \mathrm{~g}$ ), which exert the effects of clearing away heat and removing toxicity, cooling blood, and dispersing blood stasis. It is a basic prescription commonly used in TCM for the treatment of the syndrome of heat entering nutrient-blood. The clinical manifestations of SLE in the active stage mostly belong to the syndrome of exuberant heat and toxin, which coincides with the main therapeutic efficacy of XJDHT. However, the potential pharmacological mechanism of XJDHT against SLE is still unclear. The research model of "one drug-one target-one disease" cannot reflect the characteristics of TCM (multitarget and multipathway) [11].

Network pharmacology and molecular docking provide a more complete understanding of network theory and systems biology, which also help to explain the mechanism of drugs [12]. In this study, we intend to use network pharmacology to screen the active ingredients and the intersection targets of XJDHT and SLE through data analysis and bioinformatics theory. The molecular docking technique was used to fit the binding activity between the active ingredients and the core targets to provide a theoretical basis for the key mechanism of XJDHT acting on SLE. The simplified flowchart of our study is shown in Figure 1.

\section{Materials and Methods}

2.1. Collection of Potential Active Ingredients and Related Targets of XJDHT. Before conducting the screening, we found that the main ingredients of the same Chinese medicine differed in different TCM databases, and the targets of the same compounds were inconsistent in different TCM databases. Each TCM database has its advantage and disadvantage. For instance, Traditional Chinese Medicine Database and Analysis Platform (TCMSP) database [13] (https://www.tcmsp-e.com/) is the only one that provides pharmacokinetic properties, such as oral bioavailability (OB) and drug likeness (DL), whereas it only collects 499 Chinese medicines. Bioinformatics Analysis Tool for Molecular mechANism of Traditional Chinese Medicine (BATMAN-TCM) database [14] (http://bionet.ncpsb.org $. \mathrm{cn} /$ batman-tcm/) is an online bioinformatics analysis tool that contains multiple functions, but it only offers relatively few Chinese medicine ingredients. The Encyclopedia of Traditional Chinese Medicine (ETCM) database [15] (http://www.tcmip.cn/ETCM/index.php/) includes standardized information for the commonly Chinese medicines and formulas of TCM; nonetheless, only 402 herbs have been 
TABLE 1: All the potential pharmacologically active ingredients of XJDHT.

\begin{tabular}{|c|c|c|c|c|}
\hline Herb name & Molecule ID & Molecule name & $\mathrm{OB}(\%)$ & $\mathrm{DL}$ \\
\hline \multirow{7}{*}{ Bubali Cornu (Shuiniujiao, SNJ) } & MOL000987 & Cholesterol & 37.87 & 0.68 \\
\hline & MOL000054 & Arginine & 47.64 & 0.03 \\
\hline & MOL000065 & Aspartic acid & 79.74 & 0.02 \\
\hline & MOL000042 & Alanine & 87.69 & 0.01 \\
\hline & MOL001443 & 4-Guanidino-1-butanol & 26.23 & 0.01 \\
\hline & MOL006394 & Guanidine & 24 & 0 \\
\hline & MOL001921 & Lactiflorin & 49.12 & 0.80 \\
\hline \multirow{30}{*}{ Paeoniae Radix Rubra (Chishao, CS) } & MOL001924 & Paeoniflorin & 53.87 & 0.79 \\
\hline & MOL007004 & Albiflorin & 30.25 & 0.77 \\
\hline & MOL000449 & Stigmasterol & 43.83 & 0.76 \\
\hline & MOL004355 & Spinasterol & 42.98 & 0.76 \\
\hline & MOL002776 & Baicalin & 40.12 & 0.75 \\
\hline & MOL000358 & Beta-sitosterol & 36.91 & 0.75 \\
\hline & MOL000359 & Sitosterol & 36.91 & 0.75 \\
\hline & MOL006999 & Stigmast-7-en-3-ol & 37.42 & 0.75 \\
\hline & MOL005043 & Campest-5-en-3beta-ol & 37.58 & 0.71 \\
\hline & MOL007025 & Isobenzoylpaeoniflorin & 31.14 & 0.54 \\
\hline & MOL007003 & Benzoyl paeoniflorin & 31.14 & 0.54 \\
\hline & MOL007014 & 8-Debenzoylpaeonidanin & 31.74 & 0.45 \\
\hline & MOL007008 & 4-Ethyl-paeoniflorin_qt & 56.87 & 0.44 \\
\hline & MOL001002 & Ellagic acid & 43.06 & 0.43 \\
\hline & MOL007012 & 4-O-methyl-paeoniflorin_qt & 56.70 & 0.43 \\
\hline & MOL001925 & Paeoniflorin_qt & 68.18 & 0.40 \\
\hline & MOL007016 & Paeoniflorigenone & 65.33 & 0.37 \\
\hline & MOL001918 & Paeoniflorgenone & 87.59 & 0.37 \\
\hline & MOL006996 & 1-O-beta-d-glucopyranosylpaeonisuffrone_qt & 65.08 & 0.35 \\
\hline & MOL007005 & Albiflorin_qt & 48.70 & 0.33 \\
\hline & MOL006992 & $(2 \mathrm{R}, 3 \mathrm{R})$-4-methoxyl-distylin & 59.98 & 0.30 \\
\hline & MOL006994 & 1-O-beta-d-glucopyranosyl-8-o-benzoylpaeonisuffrone_qt & 36.01 & 0.30 \\
\hline & MOL007018 & 9-Ethyl-neo-paeoniaflorin A_qt & 64.42 & 0.30 \\
\hline & MOL006990 & $\begin{array}{l}\text { (1S,2S,4R)-trans-2-hydroxy-1,8-cineole-B-D- } \\
\text { glucopyranoside }\end{array}$ & 30.25 & 0.27 \\
\hline & MOL000492 & $(+)$-catechin & 54.83 & 0.24 \\
\hline & MOL007022 & EvofolinB & 64.74 & 0.22 \\
\hline & MOL002714 & Baicalein & 33.52 & 0.21 \\
\hline & MOL002883 & Ethyl oleate (NF) & 32.40 & 0.19 \\
\hline & MOL000131 & EIC & 41.9 & 0.14 \\
\hline & MOL000675 & Oleic acid & 33.13 & 0.14 \\
\hline \multirow{10}{*}{ Moutan Cortex (Mudanpi, MDP) } & MOL001746 & ELD & 31.20 & 0.14 \\
\hline & MOL000211 & Mairin & 55.38 & 0.78 \\
\hline & MOL000359 & Sitosterol & 36.91 & 0.75 \\
\hline & MOL007003 & Benzoyl paeoniflorin & 31.14 & 0.54 \\
\hline & MOL007369 & 4-O-methylpaeoniflorin_qt & 67.24 & 0.43 \\
\hline & MOL001925 & Paeoniflorin_qt & 68.18 & 0.40 \\
\hline & MOL007382 & Mudanpioside-h_qt 2 & 42.36 & 0.37 \\
\hline & MOL007384 & Paeonidanin_qt & 65.31 & 0.35 \\
\hline & MOL007374 & 5-[[5-(4-methoxyphenyl)-2-furyl]methylene]barbituric acid & 43.44 & 0.30 \\
\hline & MOL000098 & Quercetin & 46.43 & 0.28 \\
\hline
\end{tabular}


TABLE 1: Continued.

\begin{tabular}{|c|c|c|c|c|}
\hline Herb name & Molecule ID & Molecule name & $\mathrm{OB}(\%)$ & $\mathrm{DL}$ \\
\hline \multirow{13}{*}{ Dried Rehmanniae Radix (Shengdihuang, DH) } & MOL000492 & $(+)$-catechin & 54.83 & 0.24 \\
\hline & MOL000422 & Kaempferol & 41.88 & 0.24 \\
\hline & MOL000675 & Oleic acid & 33.13 & 0.14 \\
\hline & MOL000449 & Stigmasterol & 43.83 & 0.76 \\
\hline & MOL000359 & Sitosterol & 36.91 & 0.75 \\
\hline & MOL012254 & Campesterol & 37.58 & 0.71 \\
\hline & MOL002819 & Catalpol & 5.07 & 0.44 \\
\hline & MOL003735 & Aucubin & 4.17 & 0.33 \\
\hline & MOL000842 & Sucrose & 7.17 & 0.23 \\
\hline & MOL000131 & EIC & 41.9 & 0.14 \\
\hline & MOL003708 & Jioglutin D & 39.02 & 0.14 \\
\hline & MOL003706 & Jioglutin B & 90.71 & 0.13 \\
\hline & MOL000388 & Gamma-aminobutyric acid & 24.09 & 0.01 \\
\hline
\end{tabular}

collected. Traditional Chinese Medicines Integrated Database (TCMID) database (http://www.megabionet.org/tcmid/) contains 8159 kinds of Chinese medicines, which has the largest number of Chinese medicines in all databases, and each Chinese medicine ingredient retrieved is supported by relevant literature. However, it contains too little information on the targets of Chinese medicine ingredients. Therefore, we optimized the retrieval strategy. First of all, the ingredients of each Chinese medicine were retrieved from each of the four TCM databases mentioned above to obtain the main ingredients of XJDHT. Then, all the ingredients were initially screened in the TCMSP database according to $\mathrm{OB} \geq 30 \%$ and $\mathrm{DL} \geq 0.18$. For the compounds that cannot be available in the TCMSP database, the compounds with high gastrointestinal absorption and good DL were further screened out by the SwissADME database (http://www.swissadme.ch/) [15]. In addition, we found that certain compounds, although with $\mathrm{OB}<30 \%$ or $\mathrm{DL}<0.18$, have a wide range of pharmacological activities (such as oleic acid [16] and gamma-aminobutyric acid [17]) or are major ingredients of a certain Chinese medicine (such as jioglutin [18] and aspartic acid [19]), which had also been added as potential active ingredients of XJDHT. TCMSP database was used to forecast the relevant targets for potential active ingredients of XJDHT, and the names of the relevant targets were normalized through the UniProt database [20] (https://www.uniprot.org/). If TCMSP database cannot search for the compounds, the targets of the compounds would be predicted by Swiss Target Prediction (http://swisstargetprediction.ch/) to obtain all the potential targets of XJDHT, and the probability $\geq 0.12$ was used as the screening condition.

\subsection{Acquisition of Known Targets for SLE and Construction} of Venn Diagrams. "Systemic lupus erythematosus" was used as a keyword to screen in GeneCards database (http://www .genecards.org/), OMIM database (https://omim.org/), and DisGeNET database (https://www.disgenet.org/). Meanwhile, Genecards database and DisGeNET database selected scores greater than 4 and 0.1 , respectively, and all known targets were merged and removed duplicate values to obtain the union targets of SLE. Furthermore, we used Venn 2.1.0 online platform (http://bioinfogp.cnb.csic.es/tools/venny) to obtain the intersection targets of XJDHT against SLE.

2.3. Construction and Analysis of the Network of "Chinese Medicines-Active Ingredients-Intersection Targets." Cytoscape 3.8.2 is a visualization software that can show the interactions and connections between things [21]. The active ingredients of XJDHT against SLE were obtained by searching the compounds corresponding to the intersection targets. Then, we imported Chinese medicines, active ingredients, and intersection targets into Cytoscape 3.8.2 software to construct the network diagram of "Chinese medicinesactive ingredients-intersection targets." The nodes of the network diagram represent Chinese medicines, active ingredients, and intersection targets, and the edges represent their interactions. The core active ingredients in the network were analyzed according to the degree values of the ingredients.

2.4. Construction and Analysis of PPI Network and Core Network. STRING (http://string-db.org/) is a database containing the known and predicted protein-protein interactions (PPIs) [22]. The intersection targets of XJDHT and SLE were uploaded to the STRING online database platform to obtain the PPI network diagram of the intersection targets by setting the organism as "Homo sapiens" and the minimum required interaction scores $\geq 0.4$. Afterwards, the PPI network diagram and tsv format file were exported. The network topology analysis of the parameters in the PPI network was performed with the help of $\mathrm{R} 4.0 .5$ software and CytoNCA plug-in [23] of Cytoscape 3.8.2 software to finally obtain the core targets of XJDHT against SLE.

2.5. GO and KEGG Pathway Enrichment Analysis. Firstly, we installed the relevant $\mathrm{R}$ packages such as "BiocManager," "cluster Profiler," and "pathview" [24] to perform Gene Ontology (GO) and Kyoto Encyclopedia of Genes and Genomes (KEGG) pathway enrichment analysis, and the results were visually displayed through $\mathrm{R}$ 4.0.5 software. Meanwhile, GO analysis includes three aspects of molecular 


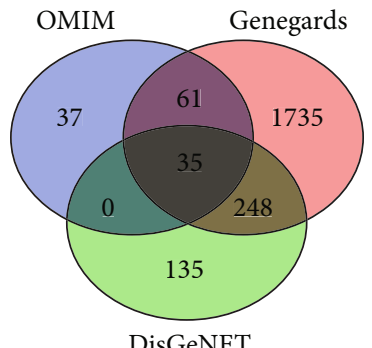

(a)

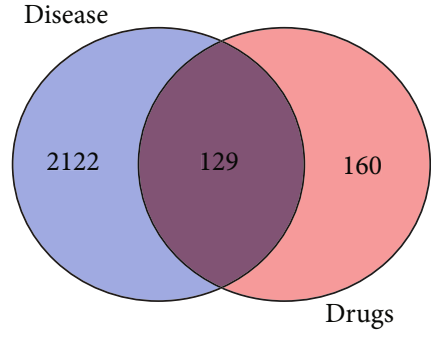

(b)

FIgURE 2: Venn diagrams. (a) Venn diagram of SLE-related genes. (b) Venn diagram of XJDHT targets and SLE targets.

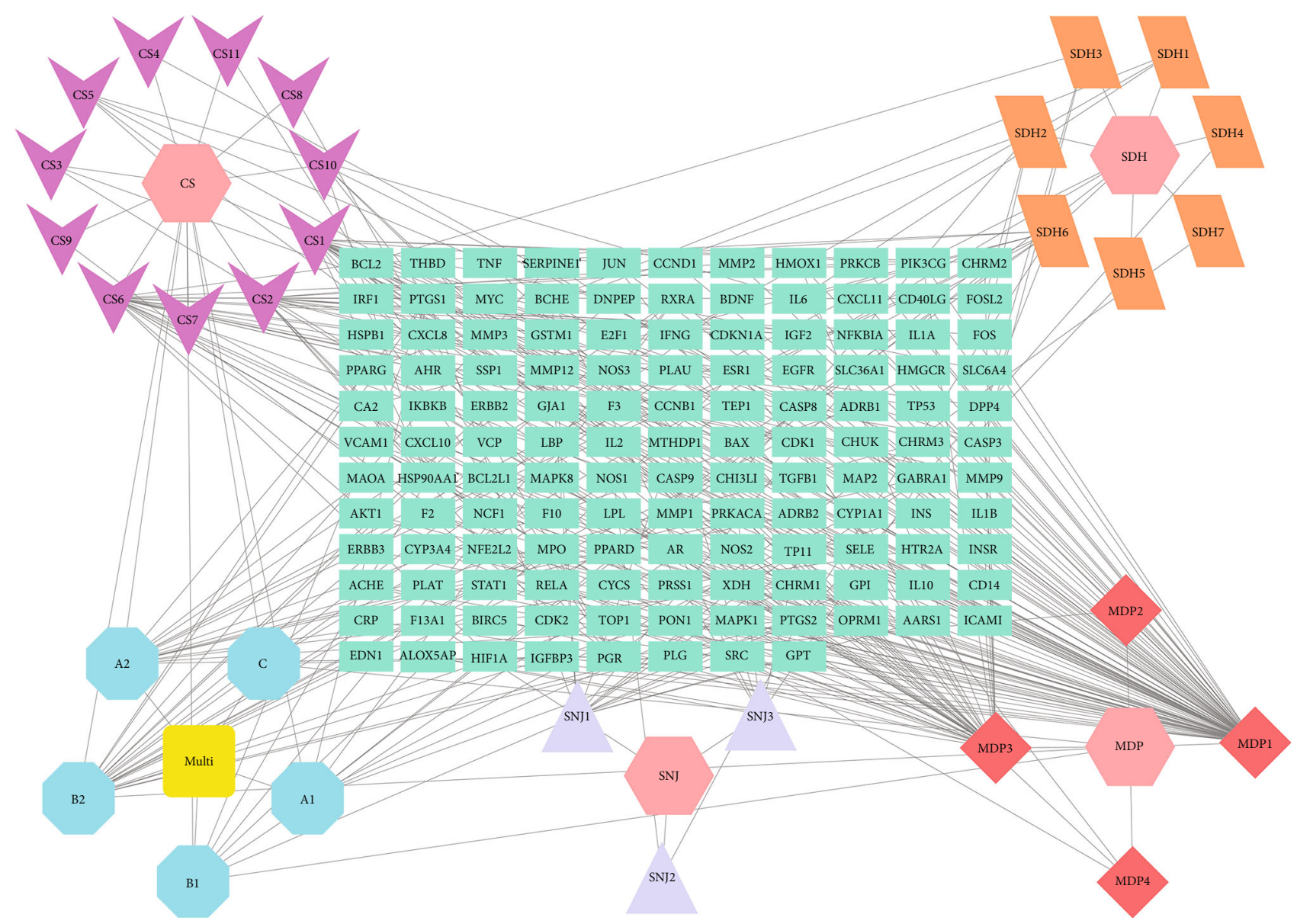

FIGURE 3: Chinese medicines-active ingredients-intersection targets network of SLE. The hexagon nodes represent all the herbs of XJDHT, the $\mathrm{V}$ nodes represent Paeoniae Radix Rubra, the diamond nodes represent Moutan Cortex, the parallelogram nodes represent Dried Rehmanniae Radix, the triangle nodes represent Bubali Cornu, the rectangle nodes represent the intersection genes, the octagon nodes represent compounds derived from multiple drugs, and the round rectangle node represents multiple compounds.

function (MF), biological process (BP), and cellular component (CC). Besides, the statistical significance of enrichment analysis was $P \leq 0.05$. Finally, the "Pathview" package was used to map the signaling pathway containing the most intersection targets.

2.6. Molecular Docking Prediction. The top three core targets in the PPI network and their corresponding compounds were selected as the receptors and the ligands for molecular docking, respectively. The crystal structure of the receptor proteins was retrieved from the RCSB PDB database (https://www.rcsb.org/), and the water molecules of the receptor proteins were removed by PyMOL 2.4.1 software. Then, the optimized receptors were imported into Auto Dock tools1.5.6 software for hydrogenation and calculation of charge, and the output results were saved as pdbqt format. The ligand 2D structures were downloaded from the PubChem database (https://pubchem.ncbi.nlm.nih.gov/) and 


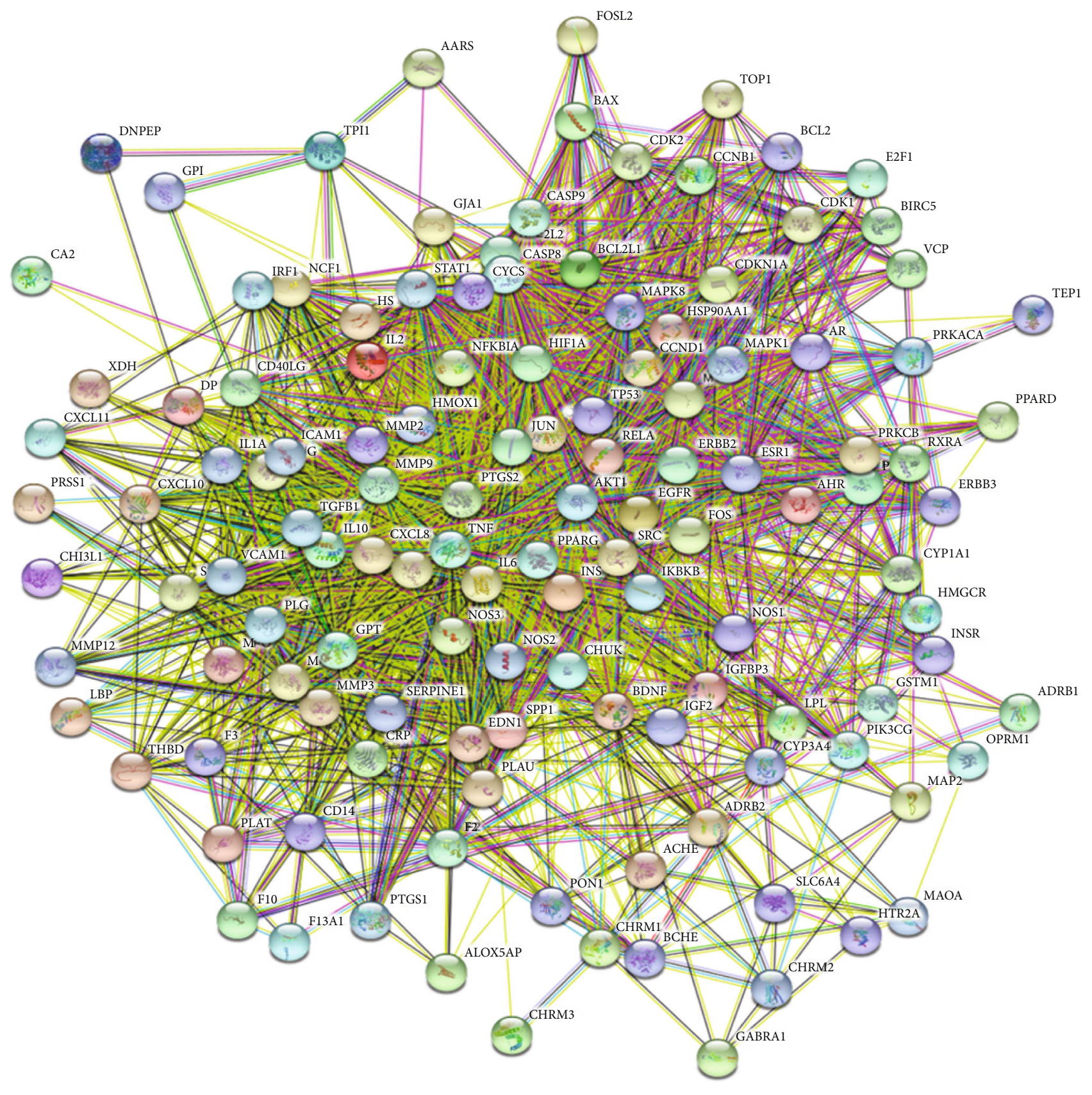

(a)

Figure 4: Continued. 


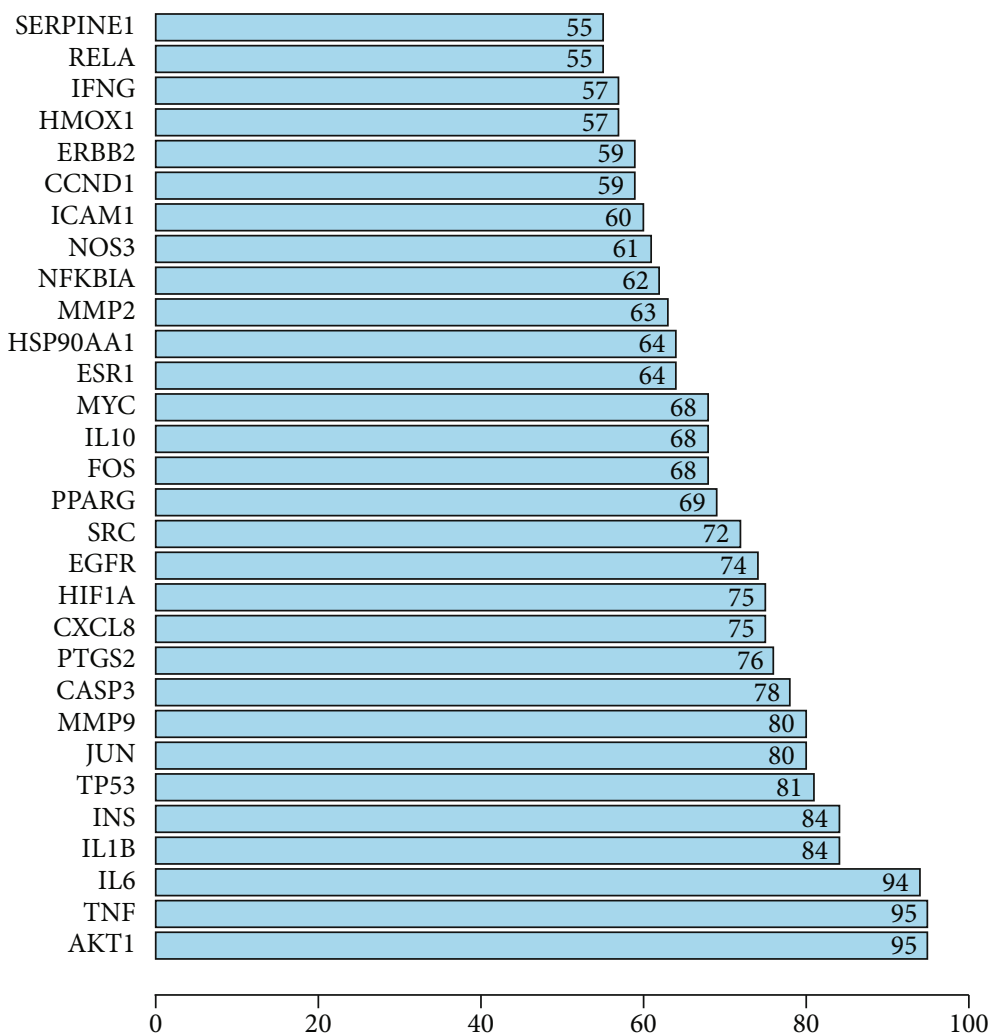

(b)

FIGURE 4: (a) PPI network of the intersection targets of XJDHT against SLE. (b) The top 20 significant genes in PPI network.

imported into the ChemBio3D Ultra 17.0 software for 3D structure conversion and optimization of the mechanical structure. The $3 \mathrm{D}$ structures of the ligands were saved as mol2 format files and imported into AutoDock Tools 1.5.6 software to save as pdbqt format. Molecular docking was performed by AutoDock vina 1.1.2 software [25], and the results were analyzed and visualized by PyMOL 2.4.1 software [26].

\section{Results}

3.1. Screening of Potential Active Ingredients and Targets of XJDHT. Through retrieving TCMSP, BATMAN-TCM, ETCM, and TCMID databases and related literature, we finally obtained 60 potential active ingredients. Although 13 of these ingredients fail to meet the screening criteria of $\mathrm{OB}$ $\geq 30 \%$ or $\mathrm{DL} \geq 0.18$, they either had a broad pharmacological activity or widely existed in multiple drugs of XJDHT, which were also considered as potential pharmacological active ingredients. The potential active ingredients of SNJ, CS, $\mathrm{SDH}$, and MDP were $6,32,10$, and 12 , respectively, and the basic information of the potential active ingredients of XJDHT is shown in Table 1. The TCMSP and Swiss Target Prediction databases were searched to acquire 693 targets for the potential active ingredients, but only 625 targets were available to exclude some targets, which were without corresponding gene names or not acting on humans. The numbers of potential targets for SNJ, CS, SDH, and MDP were 98, 199, 244 and 84, respectively. Eventually, 289 valid targets were identified after removing duplicate values.

3.2. Acquisition of XJDHT and SLE Intersection Targets. By retrieving the disease database, we obtained 133, 2079, and 416 known targets from the OMIM database, Genecards database, and DisGeNET database, respectively. A total of 2251 known targets of SLE targets were obtained after taking union and removing duplicate values. The potential targets of XJDHT were mapped to the union targets of SLE, and 129 intersection targets were available (Figure 2).

3.3. Construction and Analysis of the Network of "Chinese Medicines-Active Ingredients-Intersection Targets." A total of 30 active ingredients of XJDHT for the treatment of SLE were obtained by precisely matching the compounds corresponding to the intersection targets. "Chinese medicines-active ingredients-intersection targets" network was constructed by Cytoscape 3.8.2 software (Figure 3), which included 164 nodes and 339 edges. The degree value of a node indicates the number of nodes in the network that interact directly with the node, and the higher the degree value of a compound, the more important it is in the network. According to the results of a topological analysis, the top 6 core compounds were MDP1 (quercetin), MDP3 (kaempferol), CS6 (beta-sitosterol), CS1 (baicalein), B2 (Stigmasterol), and A2 (oleic acid) in the network, which 


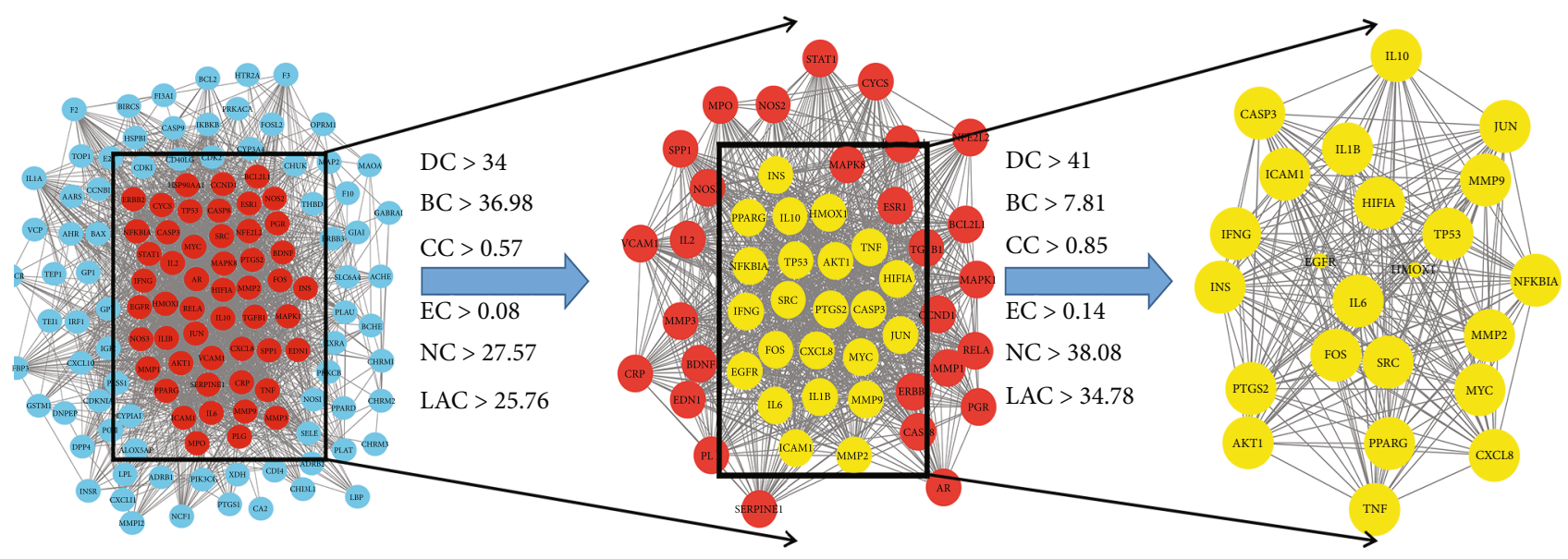

(a)

(b)

(c)

FIGURE 5: The network topology analysis of the PPI network. (a) The PPI network of the intersection targets was generated by Cytoscape, which is comprised of 124 nodes and 2324 edges. (b) The subnetwork after DC, BC, CC, EC, NC, and LAC filtration, which is comprised of 51 nodes and 1044 edges. (c) The core network after DC, BC, CC, EC, NC, and LAC filtration, which is comprised of 23 nodes and 252 edges.

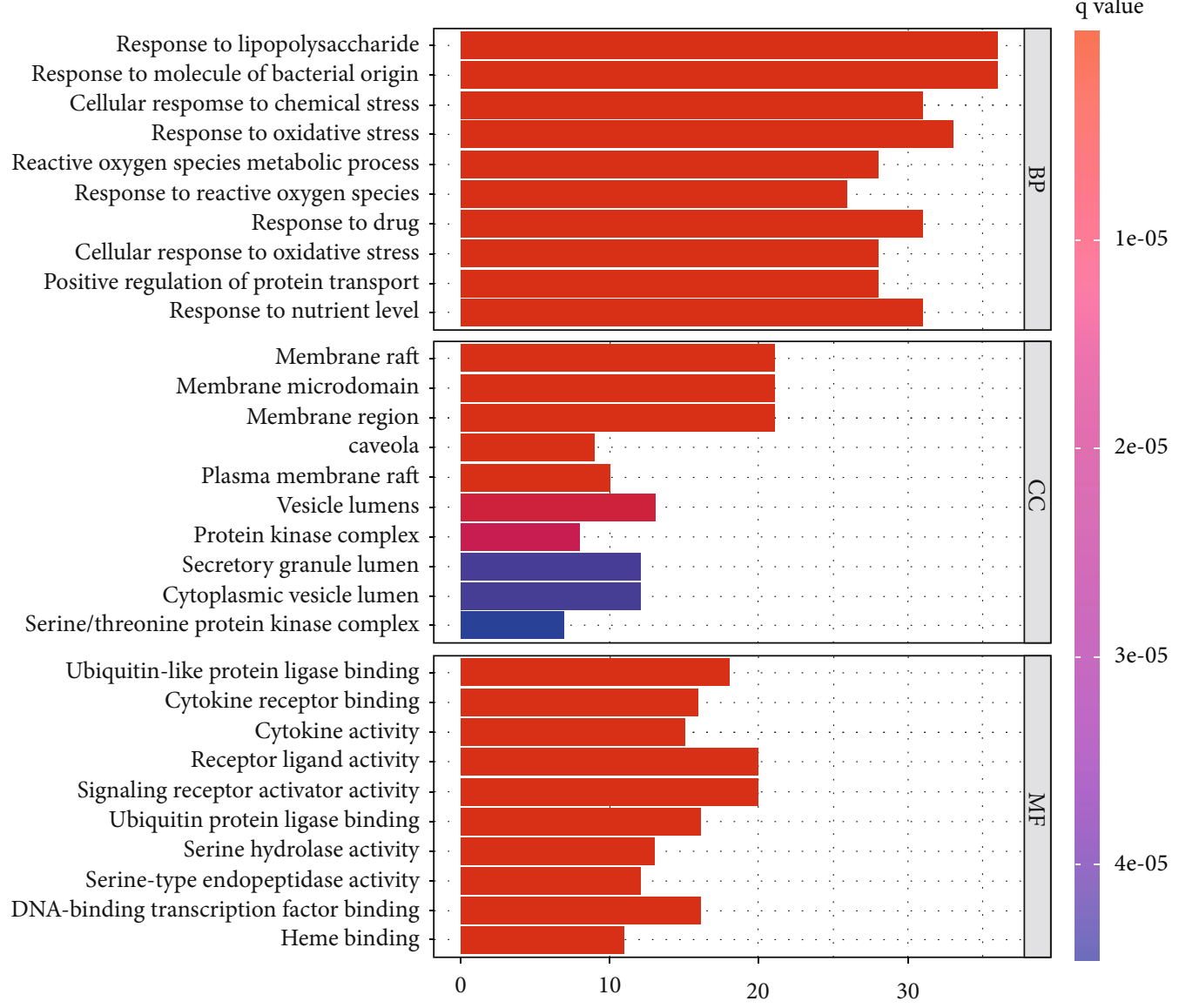

FIGURE 6: Gene Ontology terms of 129 intersection targets. The top 10 GO functional terms were selected $(P \leq 0.05)$. Abbreviations: BP: biological processes; CC: cellular component; MF: molecular function. 


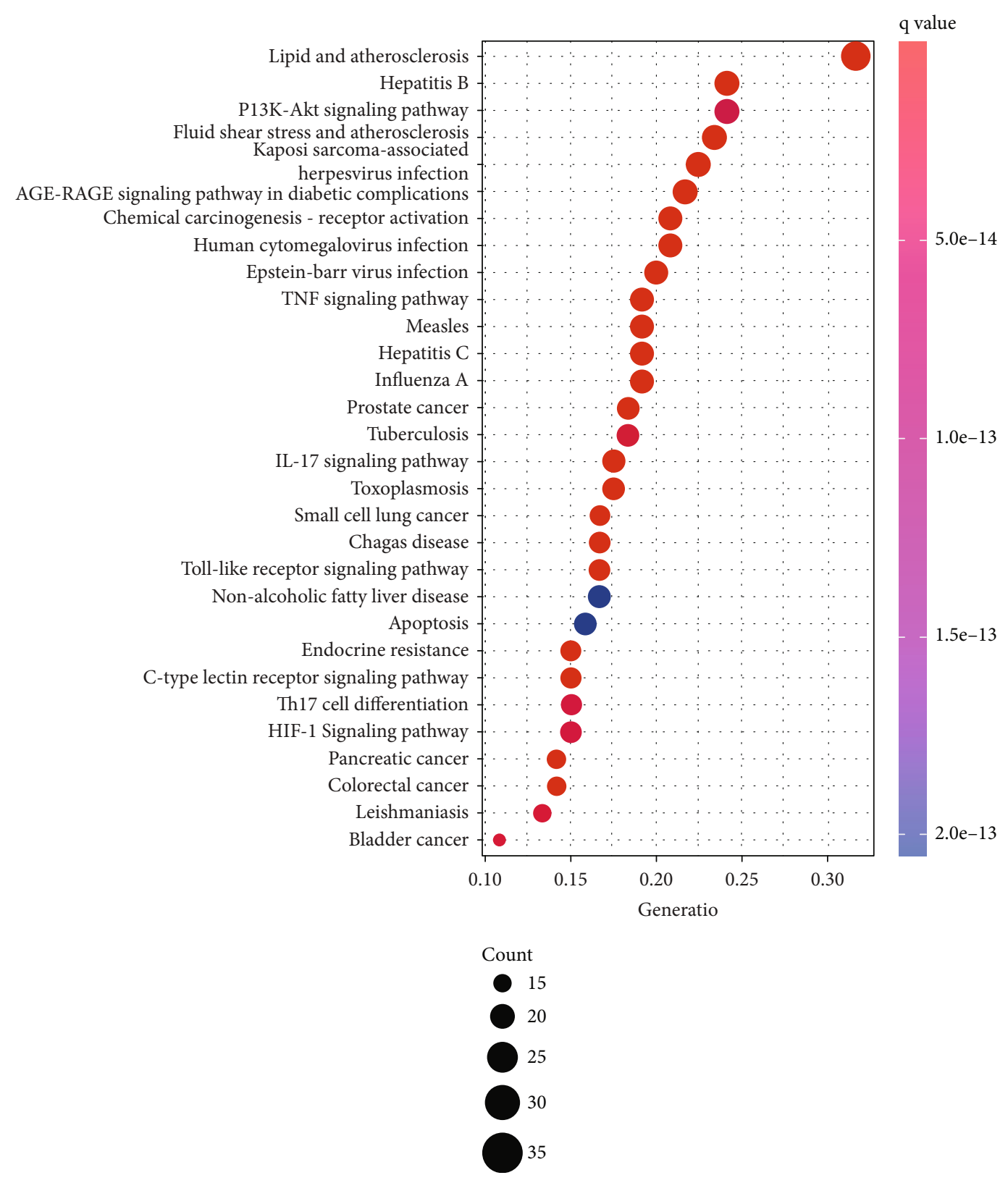

Figure 7: KEGG pathway enrichment of 129 intersection targets. The top 30 pathways were identified. Color represented $P$ value, and size of the spot represented count of genes.

indicated that the above core active compounds play an important role in the treatment of SLE.

3.4. Construction and Analysis of PPI and Core Targets Network. The intersection targets were used to construct the PPI network by STRING online database platform (Figure 4(a)). A total of 127 nodes (with 2 disconnected nodes removed) and 2324 edges were obtained, and the top 20 targets of the degree value in the PPI network were plotted as bars by $\mathrm{R} 4.0 .5$ software (Figure $4(\mathrm{~b})$ ). The core targets were screened by the CytoNCA plugin of Cytoscape 3.8.2, and the subnetwork of 23 core targets was obtained by twice filtering with scores of betweenness centrality (BC), closeness centrality (CC), degree centrality (DC), eigenvector centrality (EC), local average connectivity-based method centrality (LAC) and net- work centrality (NC) higher than the median value (Figure 5). Comparing the core targets of Figures 3 and 4, we discovered that the core targets in both figures were basically consistent. The top 10 targets in both figures were RAC-alpha serine/threonine-protein kinase (AKT1), tumor necrosis factor (TNF), interleukin-6 (IL6), interleukin-1 beta (IL1B), insulin (INS), cellular tumor antigen p53 (TP53), transcription factor AP-1(JUN), matrix metalloproteinase-9 (MMP9), caspase-3 (CASP3), and prostaglandin $\mathrm{G} / \mathrm{H}$ synthase 2 (PTGS2), which suggested that these targets were the core targets of XJDHT for the treatment of SLE.

3.5. GO and KEGG Enrichment Analysis. GO and KEGG enrichment analysis were performed through $\mathrm{R} 4.0 .5$ software, and 2535 GO terms were identified by GO enrichment 


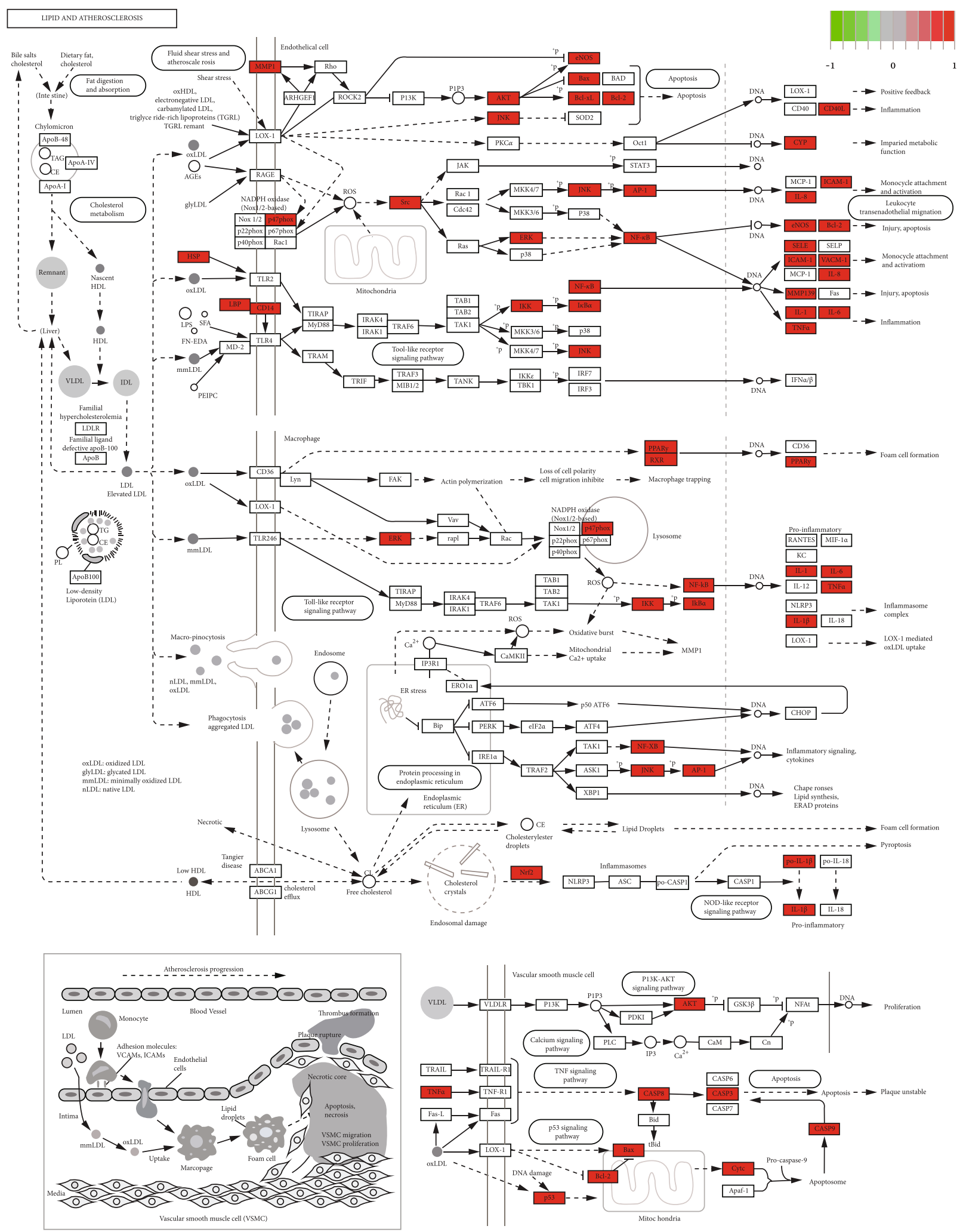

FIGURE 8: Lipid and atherosclerosis signaling pathway. Red represents the intersection targets of XJDHT against SLE in the signaling pathway.

analysis. The top 10 representative clusters of BP, CC, and MF were screened according to $\log P$ values (Figure 6). Through the pictures, we can find that the top three BP were the respond to lipopolysaccharide, respond to molecule of bacterial origin, and respond to cellular response to chemical stress, and the top three CC were the membrane raft, 
TABLE 2: The optimum model for molecular docking.

\begin{tabular}{lcc}
\hline Small molecule ligand & Receptor protein & $\begin{array}{c}\text { Binding energy/ } \\
\text { kcal.Mol }\end{array}$ \\
\hline Baicalein & AKT1 & -6.8 \\
Kaempferol & AKT1 & -6.1 \\
Quercetin & AKT1 & -6 \\
Gamma-aminobutyric acid & IL6 & -4.5 \\
Acubin & IL6 & -6.8 \\
Paeoniflorin & IL6 & -7.8 \\
Quercetin & IL6 & -7.8 \\
Aucubin & TNF & -11.8 \\
Kaempferol & TNF & -12.7 \\
Paeoniflorin & TNF & -13 \\
Quercetin & TNF & -9 \\
\hline
\end{tabular}

membrane microdomain, and membrane region, and the top three MF were the ubiquitin-like protein ligase binding, cytokine receptor binding, and cytokine activity. KEGG enrichment analysis screened out 178 signaling pathways, which were mainly enriched in the lipid and atherosclerosis, AGE-RAGE signaling pathway in diabetic complications, fluid shear stress and atherosclerosis, hepatitis B, prostate cancer, TNF signaling pathway, and IL-17 signaling pathway. Afterwards, we selected the top 30 signaling pathways with the highest significance for the visual presentation (Figure 7). Finally, the signaling pathway with the most intersection targets was plotted (Figure 8), which contained a total of 38 intersection targets.

3.6. Validation of Molecular Docking. The lower the intermolecular binding energy, the better the docking effect. The binding energy $\leq-5 \mathrm{kcal} / \mathrm{mol}$ generally indicates that the receptors and ligands have relatively good binding properties [27]. To further investigate the binding activity of the main active compounds of XJDHT and the top 3 core targets in the PPI network and their corresponding compounds was performed as molecular dockings, respectively (Table 2 and Figure 9). The binding energies of the core targets and the corresponding compounds were almost $\leq-5 \mathrm{kcal} / \mathrm{mol}$, which indicated that the affinity of the core targets and their corresponding compounds were generally high. From Table 2, we found that Paeoniflorin and TNF had the best binding ability, with the lowest binding energy $=-13 \mathrm{kcal} / \mathrm{mol}$. Through molecular docking verification, we can conclude that the core targets of XJDHT acting on SLE and the corresponding active ingredients have certain or even strong binding ability, which verifies the credibility of the network pharmacological results.

\section{Discussion}

SLE is a classic autoimmune disease that severely affects the quality of life of patients. If not treated adequately, it can accelerate multiorgan and multitissue damage. The clinical efficacy of TCM against SLE is effective, not only can it improve the symptoms of SLE patients but also is affordable for the average patients. XJDHT is a fundamental formula for the treatment of SLE with the syndrome of exuberant heat and toxin, which can alleviate the clinical symptoms of SLE patients such as butterfly-shaped erythema of the face, purple spots on the skin, high fever, irritability, and joint and muscle pain, but the molecular mechanism of action is still unclear. Related studies have proved that XJDHT can regulate inflammation, protect nerves, and inhibit apoptosis, which may treat SLE through these effects $[28,29]$.

By analyzing the "Chinese medicines-active ingredientsintersection targets" network, we found that the core active compounds in XJDHT were quercetin, kaempferol, betasitosterol, baicalein, and stigmasterol. Quercetin is a natural flavonoid with anti-inflammatory, antioxidant, immunomodulatory, and neuroprotective properties [30], which can inhibit CD4 $\mathrm{T}$ cell activation and anti-inflammatory effects of macrophages to improve the symptoms in lupus nephritis (LN) mice [31]. Dos Santos et al. [32] observed that quercetin produced nephroprotective effects in LN mice through decreasing proteinuria levels and tissue expression of IL-6 and TNF- $\alpha$. Liu et al. [33] identified that quercetin inhibited mesangial cell overproliferation in LN mice by suppressing the activation of NF- $\kappa \mathrm{B}$ signaling pathway and decreasing PTX3 expression. Kaempferol can enhance the suppressive function of regulatory $\mathrm{T}$ cells (Tregs) by reducing PIM1-mediated FOXP3 phosphorylation at S422, thereby preventing and treating SLE [34]. Macrophages are closely related to the pathogenesis of SLE [35-37]. Betasitosterol can regulate macrophage function [38]; therefore it may treat SLE by regulating macrophage function. Baicalin can adjust the balance of Nrf2/HO-1 signaling and NLRP3 expression in myeloid-derived suppressor cells (MDSCs) and reduce proteinuria and renal impairment in LN mice [39].

PPI network analysis identified AKT1, TNF, IL6, IL1B, INS, and TP53 as the core targets of XJDHT for the treatment of SLE. AKT1 is a serine-threonine protein kinase, which participates in various biological processes such as metabolism, cell survival, insulin signaling, and angiogenesis [40]. Increased AKT1 gene expression is associated with Thelper-transcription factors in SLE patients [41]. The pathogenesis of SLE is related to the activation of AKT/mTOR pathway by AKT1 downregulation of miR-633 [42]. TNF is a cytokine secreted by macrophages and an immunomodulatory molecule that can alter the balance of T-regulatory cells and participate in the pathogenesis of SLE [43]. Several studies have shown that TNF- $\alpha$ gene polymorphisms are closely related to the susceptibility to SLE [44-46]. IL6 is a cytokine with multiple biological functions in immunity and tissue regeneration, which is associated with the pathogenesis of SLE [47]. Ruchakorn et al. [48] suggested that IL-6 is related to the risk of active nonrenal SLE. Shaltout et al. [49] verified that IL6 may play a role in SLE pathogenesis through effecting on double negative $\mathrm{T}$ cells and anti-dsDNA. IL1B is a potent proinflammatory cytokine that promotes Th17 differentiation of $\mathrm{T}$ cells, and its ratio in SLE patients with disease activity is less than in SLE patients with moderate disease [50]. TP53 is a central regulator of apoptosis [51], and its rs1042522G/C polymorphism is significantly 


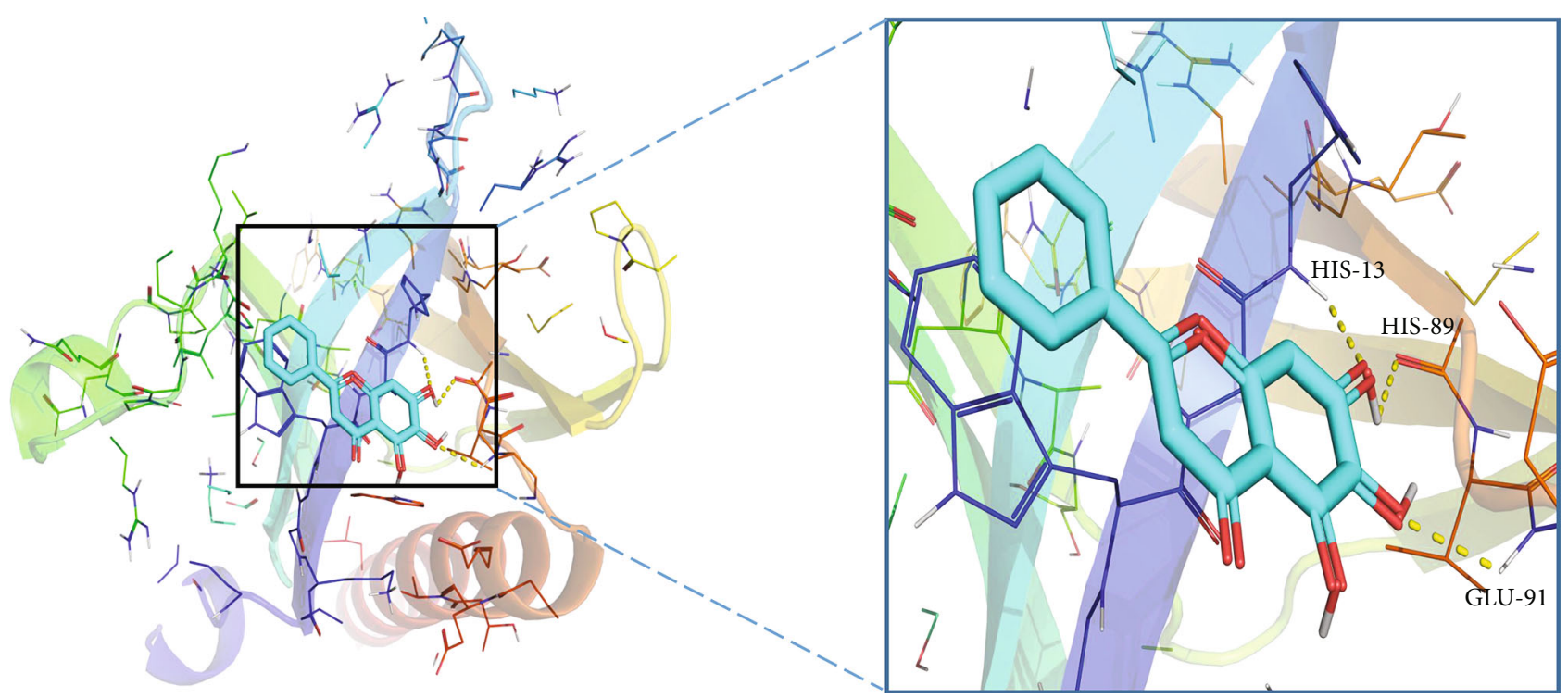

(a)

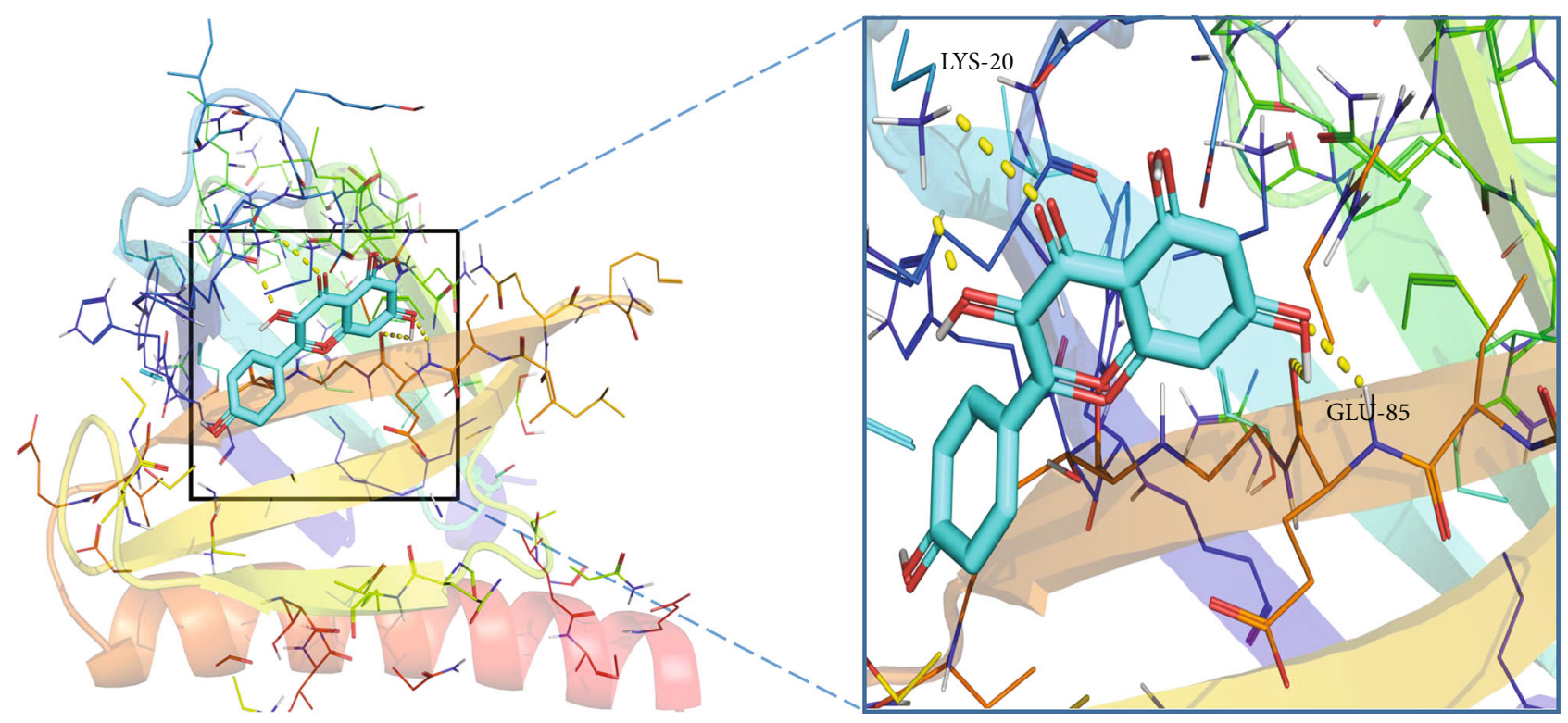

(b)

FIgURE 9: Continued. 


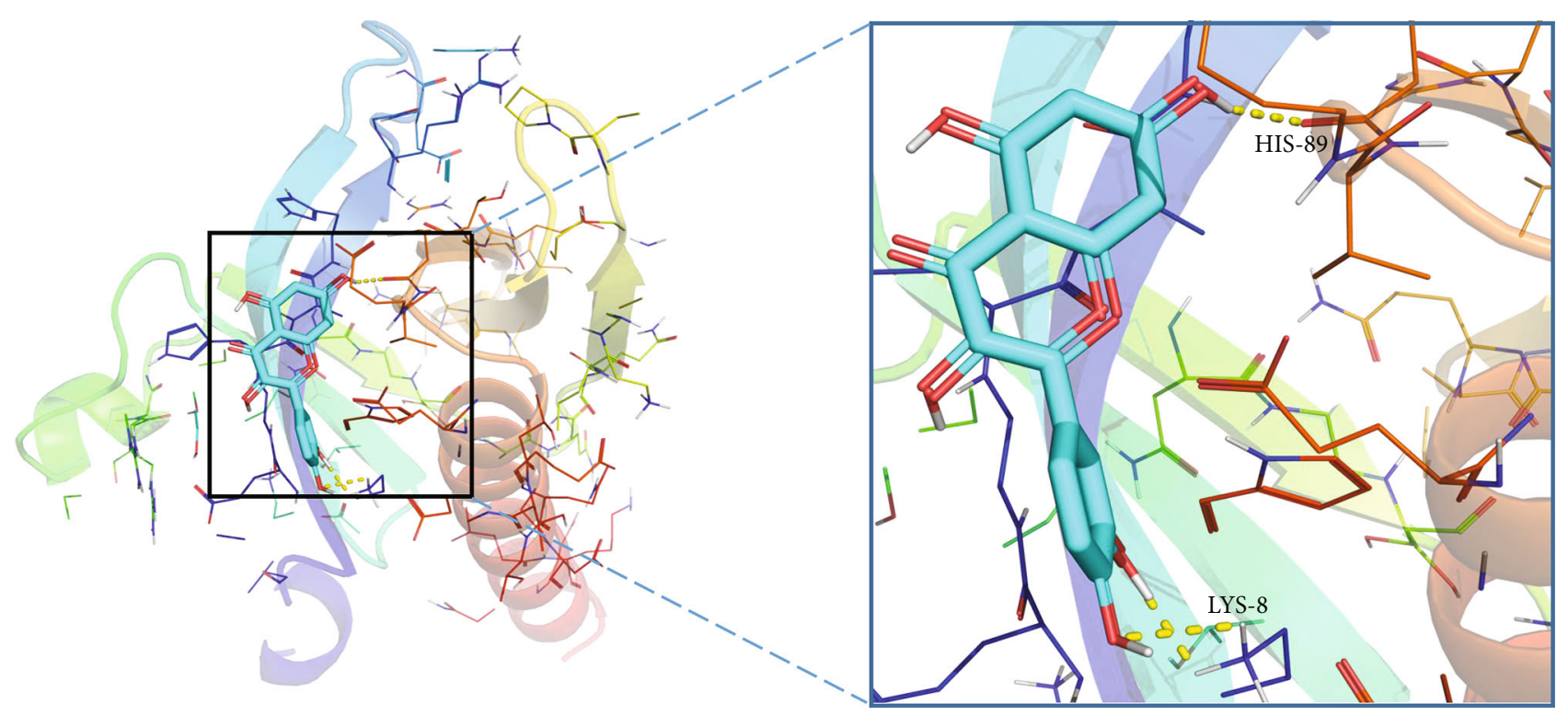

(c)

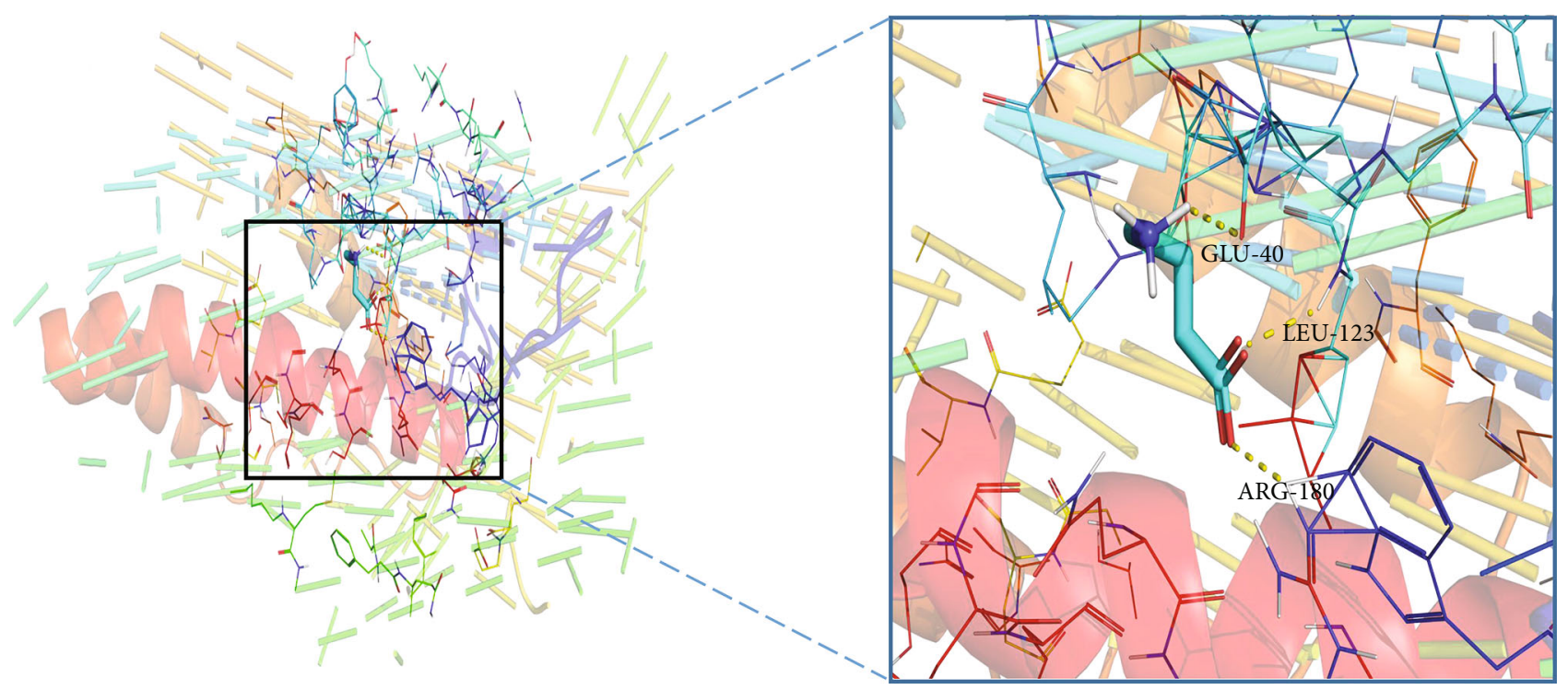

(d)

FIgURE 9: Continued. 


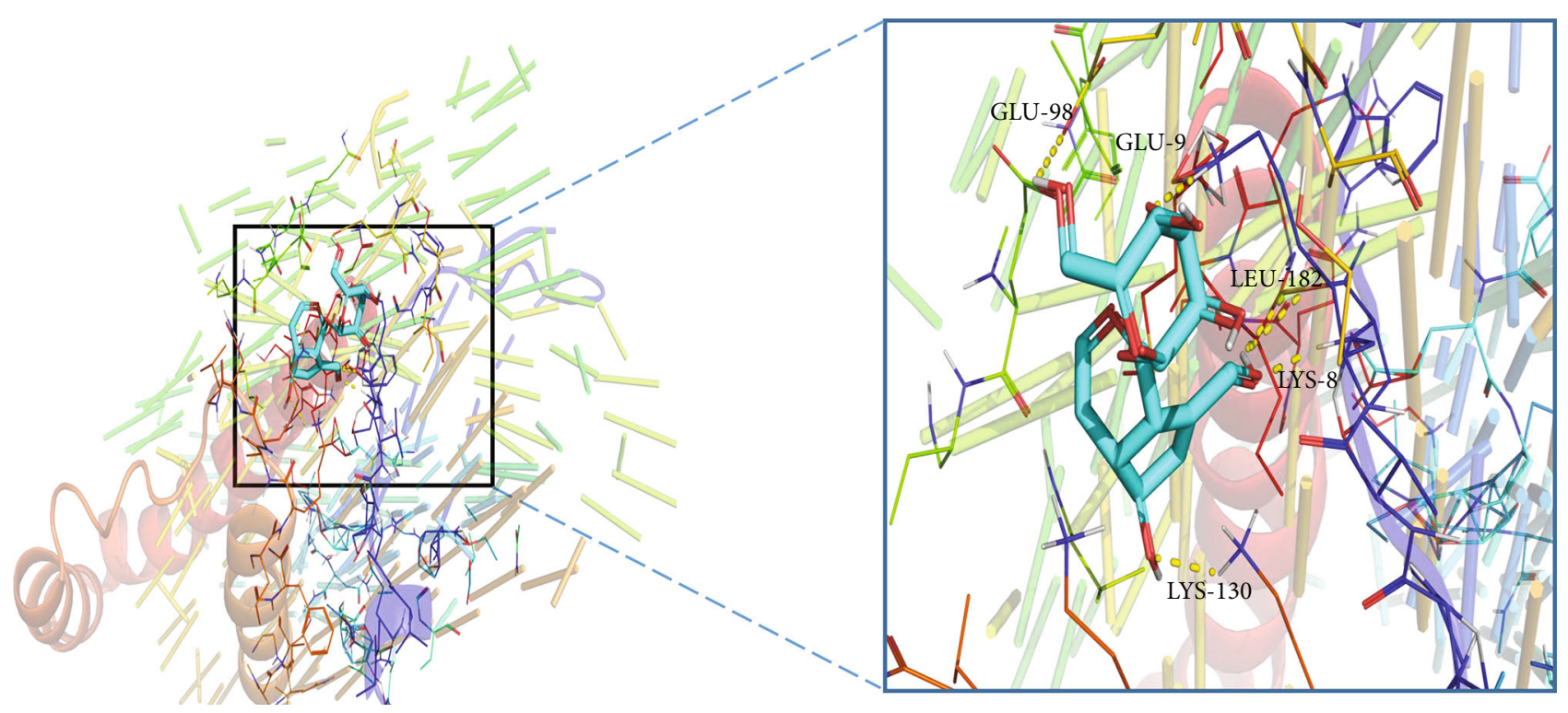

(e)

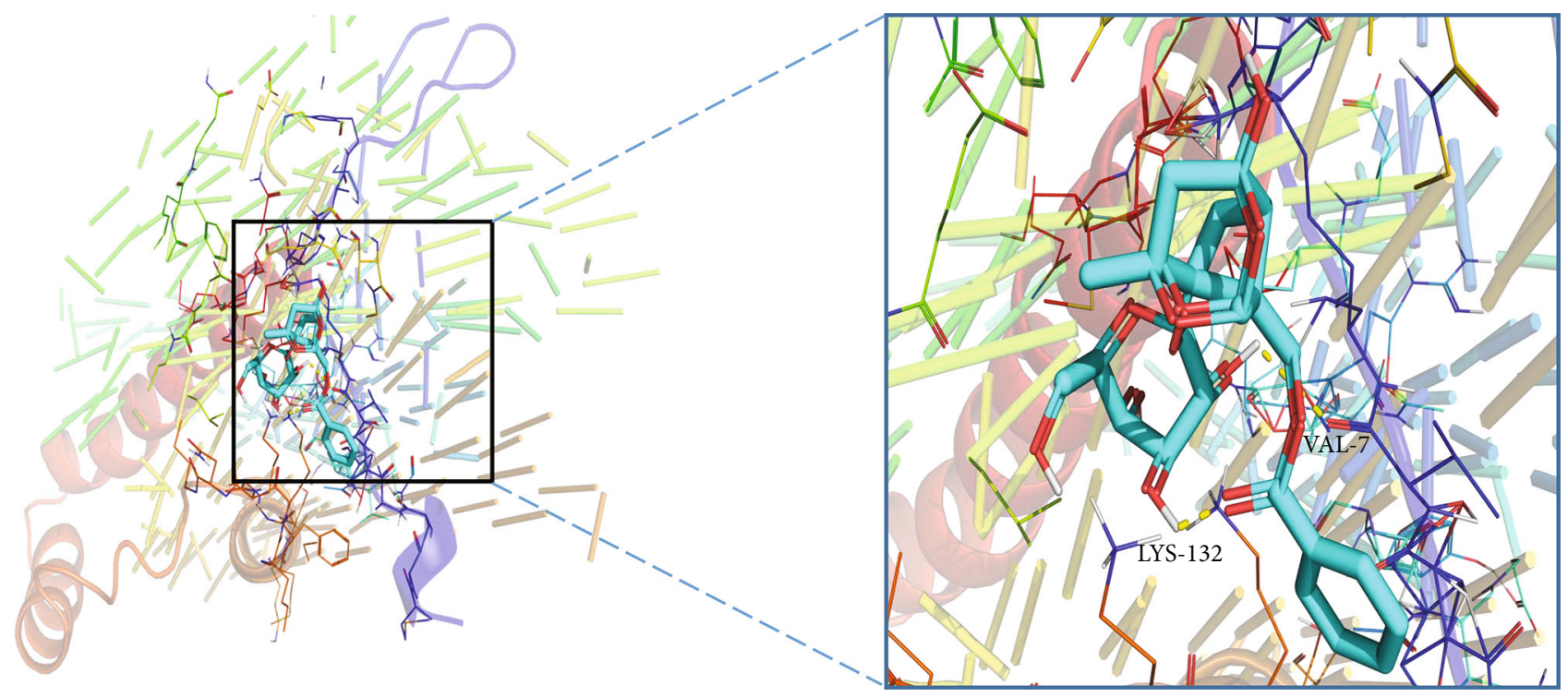

(f)

Figure 9: Continued. 


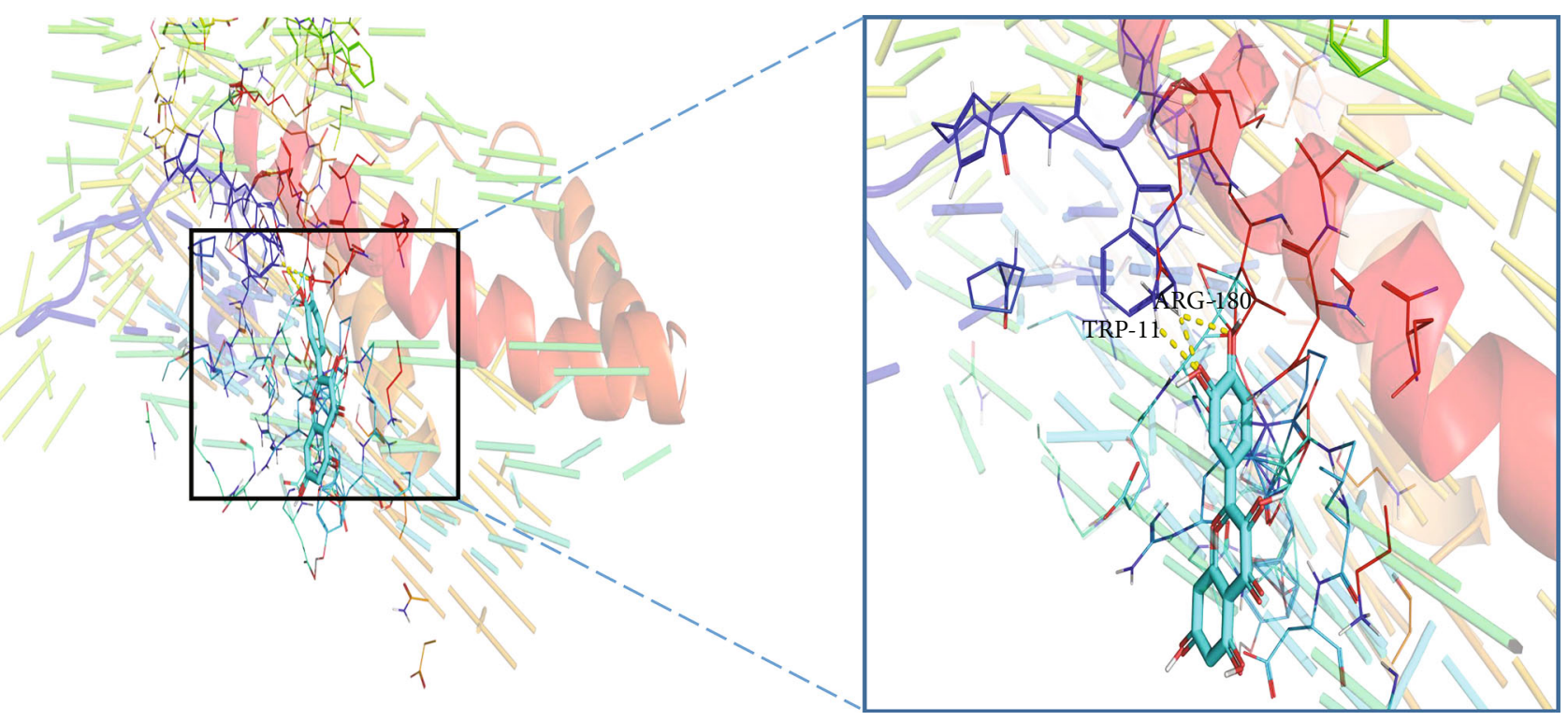

(g)

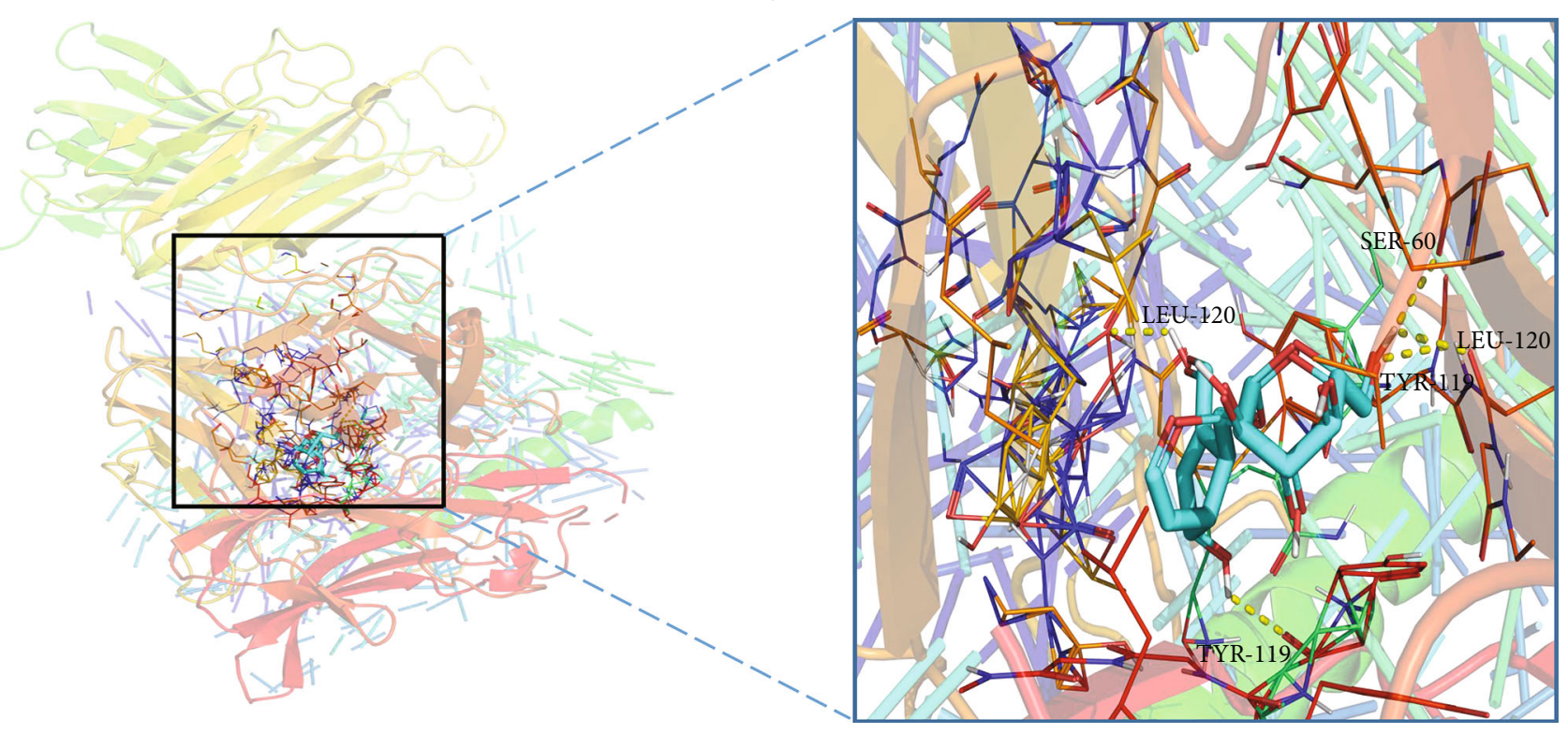

(h)

Figure 9: Continued. 


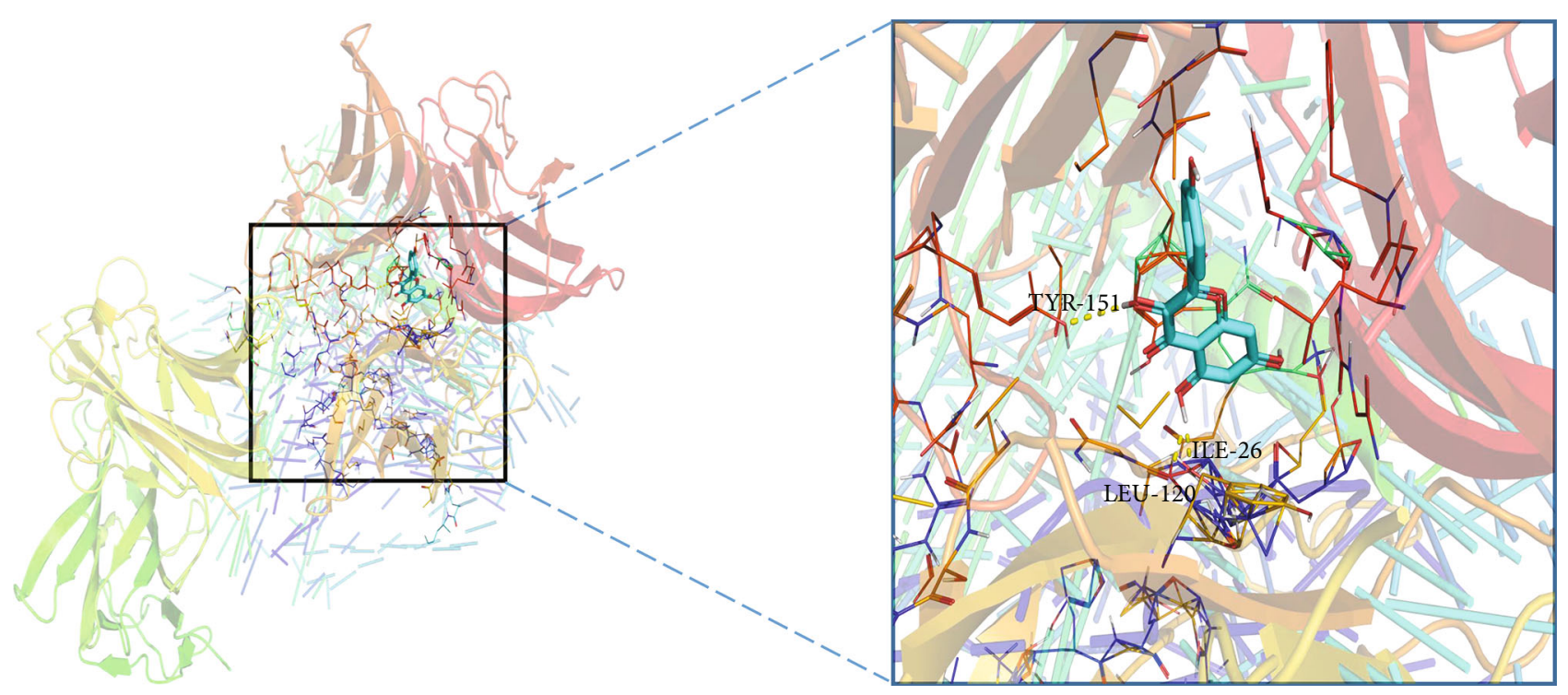

(i)

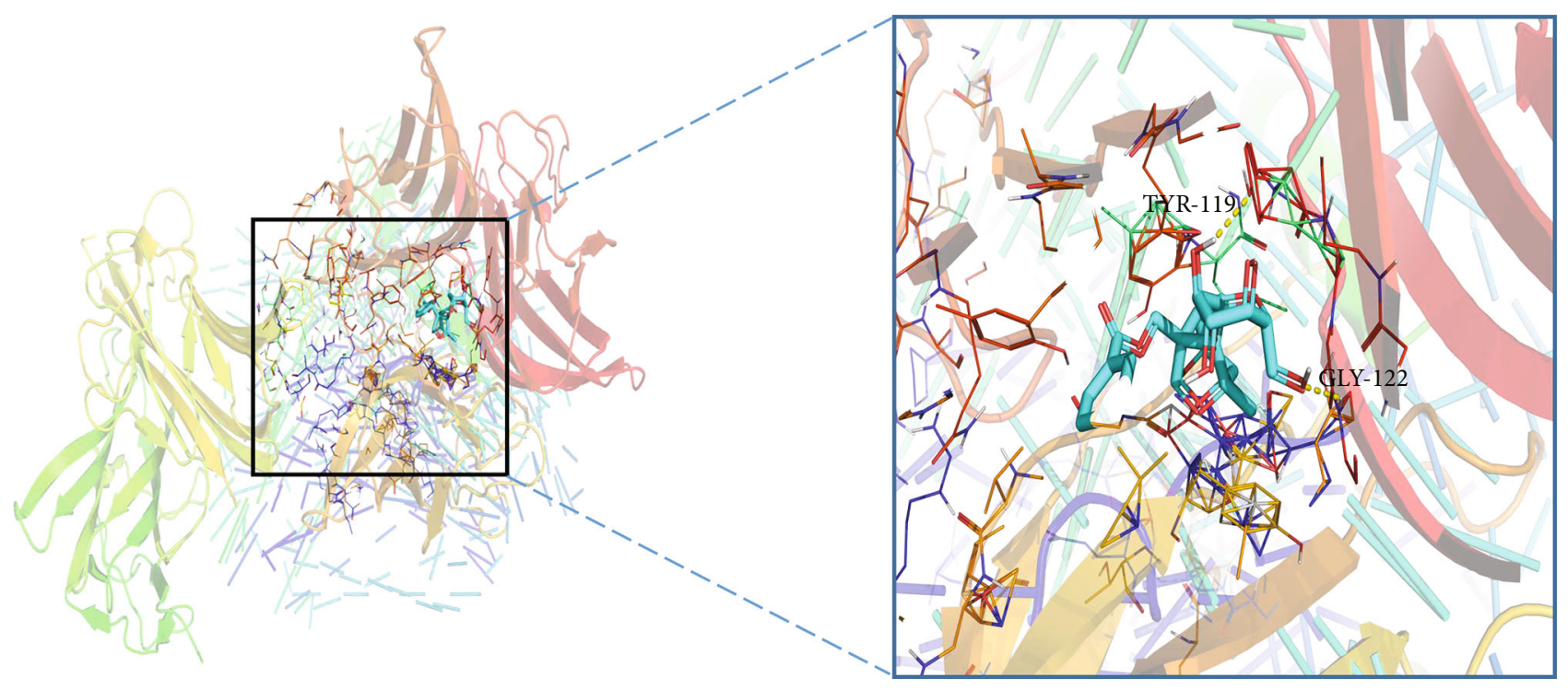

(j)

FIgure 9: Continued. 


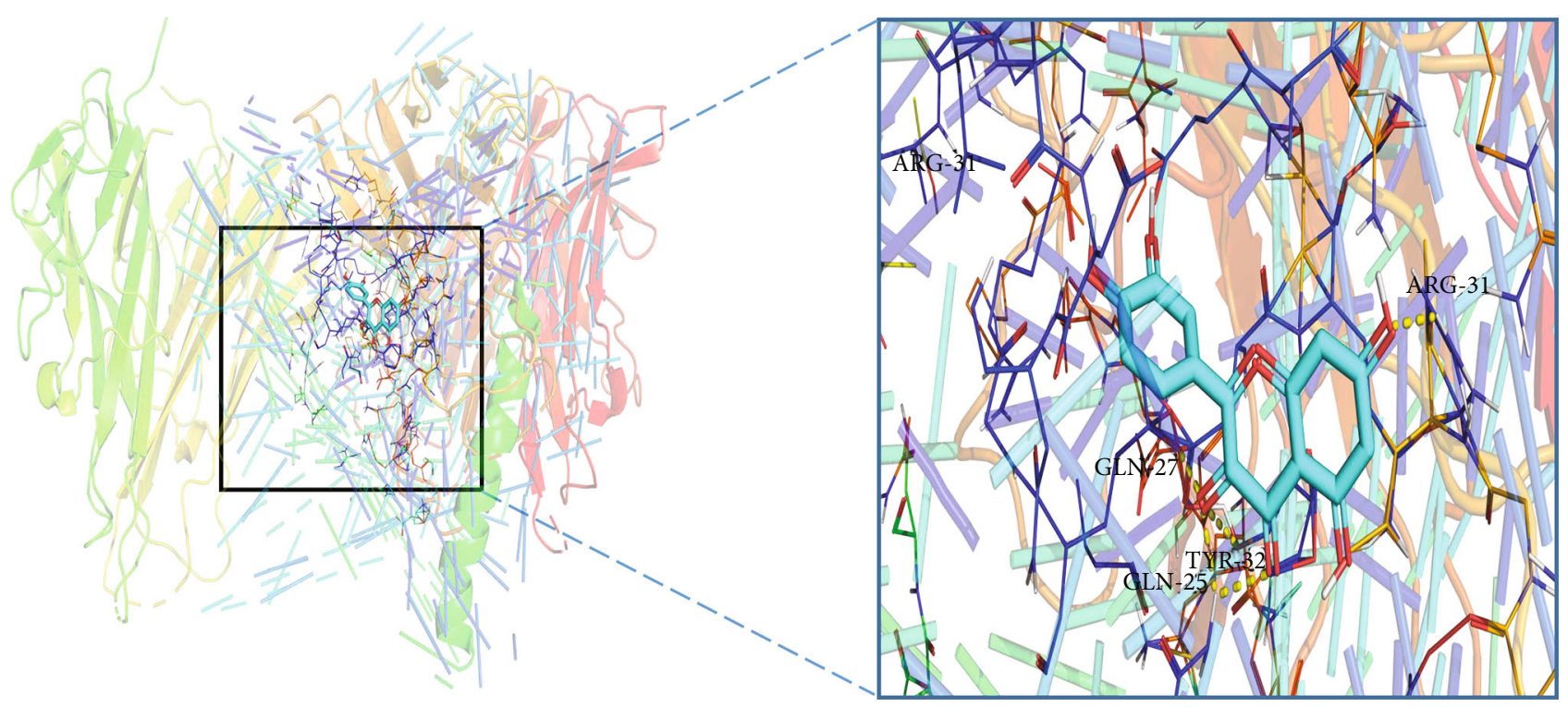

(k)

FIGURE 9: Molecular dockings of the top 3 core targets and their corresponding compounds. (a) AKT1 and baicalein. (b) AKT1 and kaempferol. (c) AKT1 and quercetin. (d) IL6 and gamma-aminobutyric acid. (e) IL6 and acubin. (f) IL6 and paeoniflorin. (g) IL6 and quercetin. (h) TNF and aucubin. (i) TNF and kaempferol. (j) TNF and paeoniflorin. (k) TNF and quercetin.

associated with SLE in Chinese Han population [52]. Teng et al. [53] revealed that the high expression of miR-564 in patients with SLE promoted differentiation of dendritic cells by negatively regulating TP53 expression.

Conducting GO analysis on the intersection genes, we found that XJDHT exerted its effects on SLE through the response to lipopolysaccharide, response to oxidative stress, cytokine receptor binding, and cytokine activity. KEGG enrichment analysis demonstrated that the main pathways involve in the lipid and atherosclerosis, AGE-RAGE signaling pathway in diabetic complications, and fluid shear stress and atherosclerosis, and each of the each pathway contains multiple targets. The number of intersection targets of the pathway indicates the importance of the pathway. The pathway with the highest number of genes was the lipid and atherosclerosis, which contains 38 targets. It mainly involves phosphoinositide 3-kinase- (PI3K-) AKT, TNF, c-Jun N-terminal kinase (JNK), and mitogen-activated protein kinase 1/3 (ERK) pathway, and their functions are mainly focused on the proinflammatory, inflammatory signal cvtokines, proliferation, injury, and apoptosis processes. PI3K-AKT signaling pathway plays an important role in cellular proliferation, and AKT expression is increased in SLE patients [54]. Dysregulation of the PI3K-AKT pathway in the MRL/lpr lupus mice was particularly prominent [55]. Furthermore, upregulation of FoxO3a expression by PI3KAKT pathway attenuated the progression of nephritis in LN mice [56]. JNK and ERK often interact with each other and are both associated with apoptosis [57], and their activity is positively correlated with SLE activity [58]. The reduction of ERK/JNK ratio can predict the severity of organ damage in SLE patients [59].

Molecular docking results demonstrated that the docking energies of the core targets and their corresponding active ingredients were almost all less than $-5 \mathrm{kcal} / \mathrm{mol}$, which indicates that the active ingredients of XJDHT have the good binding activity to the core targets.

In this study, the molecular mechanism of XJDHT for the treatment of SLE was elucidated through the screening of the active ingredients and the enrichment analysis of the intersection targets. The active ingredients of XJDHT (such as quercetin and kaempferol) act on the core targets (like AKT1 and TNF), which affect multiple signaling pathways to treat SLE. Molecular docking initially validated the interaction patterns of the major active ingredients with the core targets. However, there are still some limitations. The present study lacks the consideration of the interactions between the ingredients and the content of each ingredient. In addition, the results should be undertaken by further experiments.

\section{Conclusions}

In conclusion, this study preliminarily revealed the pharmacological effects of XJDHT against SLE through network pharmacology and molecular docking methods. A total of 30 active ingredients from XJDHT were discovered to be associated with SLE, and 23 corresponding genes were identified as core targets. The molecular mechanism of XJDHT acting on SLE is intimately related to the key GO terms and KEGG signaling pathways, such as the react to lipopolysaccharide, membrane raft, ubiquitin-like protein ligase binding, lipid, and atherosclerosis, AGE-RAGE signaling pathway in diabetic complications, fluid shear stress, and atherosclerosis. XJDHT may achieve the treatment for SLE by inhibiting the proinflammatory factors, inflammatory signal cvtokines, proliferation, injury, and apoptosis processes. This study provides the foundation for further research on the mechanism of XJDHT in the treatment of SLE. 


\section{Data Availability}

The data used to support the findings of this study are included within the article and supplementary information files.

\section{Conflicts of Interest}

The authors declare that there are no conflicts of interest regarding the publication of this paper.

\section{Acknowledgments}

The authors thank the members of their laboratory and their collaborators for their research work. This article was funded by the National Science Foundation of China (nos. 81760840 and 82160874).

\section{Supplementary Materials}

Table S1: all the potential targets of XJDHT. Table S2: known SLE-related targets. Table S3: XJDHT shared 129 intersection targets with known SLE-related targets. Table S4: protein-protein interaction information of XJDHT targets for SLE in STRING 11.0. Table S5: results for GO pathway enrichment analysis. Table S6: results for KEGG pathway enrichment analysis. (Supplementary Materials)

\section{References}

[1] M. Koo, "Systemic lupus erythematosus research: a bibliometric analysis over a 50-Year Period," International Journal of Environmental Research and Public Health, vol. 18, no. 13, 2021.

[2] F. Rees, M. Doherty, M. J. Grainge, P. Lanyon, and W. Zhang, "The worldwide incidence and prevalence of systemic lupus erythematosus: a systematic review of epidemiological studies," Rheumatology, vol. 56, no. 11, pp. 1945-1961, 2017.

[3] O. P. Rekvig, "Autoimmunity and SLE: factual and semantic evidence-based critical analyses of definitions, etiology, and pathogenesis," Frontiers in Immunology, vol. 11, 2020.

[4] L. Chen, Y. F. Wang, L. Liu et al., "Genome-wide assessment of genetic risk for systemic lupus erythematosus and disease severity," Human Molecular Genetics, vol. 29, no. 10, pp. 1745-1756, 2020.

[5] V. R. Moulton, "Sex hormones in acquired immunity and autoimmune disease," Frontiers in Immunology, vol. 9, 2018.

[6] A. Fanouriakis, M. Kostopoulou, A. Alunno et al., "Update of the EULAR recommendations for the management of systemic lupus erythematosus," Annals of the Rheumatic Diseases, vol. 78, no. 6, pp. 736-745, 2019.

[7] B. Lee, S. Hong, M. Kim et al., "Lycii radicis cortex inhibits glucocorticoid-induced bone loss by downregulating Runx2 and BMP-2 expression," International Journal of Molecular Medicine, vol. 48, no. 2, p. 155, 2021.

[8] X. Li, Z. He, L. Ru et al., "Efficacy and safety of Qinghao Biejia decoction in the treatment of systemic lupus erythematosus: a systematic review and meta-analysis," Frontiers in pharmacology, vol. 12, 2021.

[9] Y. C. Ma, C. C. Lin, C. I. Li, J. H. Chiang, T. C. Li, and J. G. Lin, "Traditional Chinese medicine therapy improves the survival of systemic lupus erythematosus patients," Seminars in Arthritis and Rheumatism, vol. 45, no. 5, pp. 596-603, 2016.

[10] L. Dai, K. K. Chan, J. C. Mao et al., "Modified Zhibai Dihuang pill, a traditional Chinese medicine formula, on steroid withdrawal in systemic lupus erythematosus: a systematic review and meta-analysis," Journal of Integrative Medicine, vol. 18, no. 6, pp. 478-491, 2020.

[11] Z. Lin, L. Zhang, and D. Tao, "Investigation on the mechanism of Qubi formula in treating psoriasis based on network pharmacology," Evidence-Based Complementary and Alternative Medicine, vol. 2020, Article ID 4683254, 13 pages, 2020.

[12] R. Al Mahmud, R. A. Najnin, and A. H. Polash, "A survey of web-based chemogenomic data resources," Methods in Molecular Biology, vol. 1825, pp. 3-62, 2018.

[13] J. Ru, P. Li, J. Wang et al., "TCMSP: a database of systems pharmacology for drug discovery from herbal medicines," Journal of Cheminformatics, vol. 6, p. 13, 2014.

[14] Z. Liu, F. Guo, Y. Wang et al., "BATMAN-TCM: a bioinformatics analysis tool for molecular mechanism of traditional Chinese medicine," Scientific Reports, vol. 6, pp. 1-11, 2016.

[15] H. Y. Xu, Y. Q. Zhang, Z. M. Liu et al., "ETCM: an encyclopaedia of traditional Chinese medicine," Nucleic Acids Research, vol. 47, no. D1, pp. D976-D982, 2019.

[16] P. Y. Gong, Y. S. Tian, Y. J. Guo et al., "Comparisons of antithrombosis, hematopoietic effects and chemical profiles of dried and rice wine-processed Rehmanniae Radix extracts," Journal of Ethnopharmacology, vol. 231, pp. 394-402, 2019.

[17] G. Martín-Ezquerra, M. Sánchez-Regaña, and P. Umbert-Millet, "Optimization of narrow-band uvb with a $5 \%$ oleic acid cream in the treatment of psoriasis," Journal of Drugs in Dermatology, vol. 6, no. 3, pp. 290-292, 2007.

[18] R. Inotsuka, K. Uchimura, A. Yamatsu, M. Kim, and Y. Katakura, " $\gamma$-Aminobutyric acid (GABA) activates neuronal cells by inducing the secretion of exosomes from intestinal cells," Food \& Function, vol. 11, no. 10, pp. 9285-9290, 2020.

[19] A. Daina, O. Michielin, and V. Zoete, "SwissADME: a free web tool to evaluate pharmacokinetics, drug-likeness and medicinal chemistry friendliness of small molecules," Scientific Reports, vol. 7, p. 42717, 2017.

[20] C. H. Chao, J. L. Hsu, M. F. Chen et al., "Anti-hypertensive effects of Radix Rehmanniae and its active ingredients," Natural Product Research, vol. 34, no. 11, pp. 1547-1552, 2020.

[21] The UniProt Consortium, "UniProt: the universal protein knowledgebase in 2021," Nucleic Acids Research, vol. 49, no. D1, pp. D480-D489, 2021.

[22] P. Shannon, A. Markiel, O. Ozier et al., "Cytoscape: a software environment for integrated models of biomolecular interaction networks," Genome Research, vol. 13, no. 11, pp. 24982504, 2003.

[23] H. V. Cook, N. T. Doncheva, D. Szklarczyk, C. Von Mering, and L. J. Jensen, "Viruses.STRING: a virus-host protein-protein interaction database," Viruses, vol. 10, no. 10, p. 519, 2018.

[24] Y. Tang, M. Li, J. Wang, Y. Pan, and F. X. Wu, "CytoNCA: a cytoscape plugin for centrality analysis and evaluation of protein interaction networks," Bio Systems, vol. 127, pp. 67-72, 2015.

[25] G. Yu, L. G. Wang, Y. Han, and Q. Y. He, "clusterProfiler: an R package for comparing biological themes among gene clusters," OMICS, vol. 16, no. 5, pp. 284-287, 2012.

[26] G. Bitencourt-Ferreira, V. O. Pintro, and W. F. de Azevedo Jr., "Docking with AutoDock4," Methods in Molecular Biology, vol. 2053, pp. 125-148, 2019. 
[27] N. T. Nguyen, T. H. Nguyen, T. N. H. Pham et al., "Autodock Vina adopts more accurate binding poses but Autodock4 forms better binding affinity," Journal of Chemical Information and Modeling, vol. 60, no. 1, pp. 204-211, 2020.

[28] X. Fei, X. Zhang, Q. Wang et al., "Xijiao Dihuang decoction alleviates ischemic brain injury in MCAO rats by regulating inflammation, neurogenesis, and angiogenesis," EvidenceBased Complementary and Alternative Medicine, vol. 2018, 12 pages, 2018.

[29] Y. M. Liu, L. L. Zhu, R. Li et al., "Xijiao Dihuang decoction () and Rehmannia glutinosa Libosch. protect mice against lipopolysaccharide and tumor necrosis factor alpha-induced acute liver failure," Chinese Journal of Integrative Medicine, vol. 25, no. 6, pp. 446-453, 2019.

[30] P. Shen, W. Lin, X. Deng et al., "Potential implications of quercetin in autoimmune diseases," Frontiers in Immunology, vol. 12, article 689044, 2021.

[31] W. Li, H. Li, M. Zhang et al., "Quercitrin ameliorates the development of systemic lupus erythematosus-like disease in a chronic graft-versus-host murine model," American Journal of Physiology. Renal Physiology, vol. 311, no. 1, pp. F217F226, 2016.

[32] M. Dos Santos, P. T. Poletti, G. Favero et al., "Protective effects of quercetin treatment in a pristane-induced mouse model of lupus nephritis," Autoimmunity, vol. 51, no. 2, pp. 69-80, 2018.

[33] Y. Liu, C. Yu, K. Ji et al., "Quercetin reduces TNF- $\alpha$-induced mesangial cell proliferation and inhibits PTX3 production: involvement of NF- $\kappa \mathrm{B}$ signaling pathway," Phytotherapy Research, vol. 33, no. 9, pp. 2401-2408, 2019.

[34] F. Lin, X. Luo, A. Tsun, Z. Li, D. Li, and B. Li, "Kaempferol enhances the suppressive function of Treg cells by inhibiting FOXP3 phosphorylation," International Immunopharmacology, vol. 28, no. 2, pp. 859-865, 2015.

[35] J. Orme and C. Mohan, "Macrophages and neutrophils in SLEan online molecular catalog," Autoimmunity Reviews, vol. 11, no. 5, pp. 365-372, 2012.

[36] N. I. Maria and A. Davidson, "Renal macrophages and dendritic cells in SLE nephritis," Current rheumatology reports, vol. 19, no. 12, p. 81, 2017.

[37] C. B. Crayne, S. Albeituni, K. E. Nichols, and R. Q. Cron, “The immunology of macrophage activation syndrome," Frontiers in Immunology, vol. 10, p. 119, 2019.

[38] R. Liu, D. Hao, W. Xu et al., “ $\beta$-Sitosterol modulates macrophage polarization and attenuates rheumatoid inflammation in mice," Pharmaceutical Biology, vol. 57, no. 1, pp. 161-168, 2019.

[39] D. Li, G. Shi, J. Wang et al., "Baicalein ameliorates pristaneinduced lupus nephritis via activating Nrf2/HO-1 in myeloid-derived suppressor cells," Arthritis Research \& Therapy, vol. 21, no. 1, p. 105, 2019.

[40] I. Hers, E. E. Vincent, and J. M. Tavaré, "Akt signalling in health and disease," Cellular Signalling, vol. 23, no. 10, pp. 1515-1527, 2011.

[41] S. Garcia-Rodriguez, J. L. Callejas-Rubio, N. Ortego-Centeno et al., "Altered AKT1 and MAPK1 gene expression on peripheral blood mononuclear cells and correlation with T-helpertranscription factors in systemic lupus erythematosus patients," Mediators of Inflammation, vol. 2012, Article ID 495934, 2012.

[42] S. Chen, Y. Wang, H. Qin et al., "Downregulation of miR-633 activated AKT/mTOR pathway by targeting AKT1 in lupus CD4+ T cells," Lupus, vol. 28, no. 4, pp. 510-519, 2019.
[43] L. J. Zhu, X. Yang, and X. Q. Yu, "Anti-TNF-alpha therapies in systemic lupus erythematosus," Journal of Biomedicine \& Biotechnology, vol. 2010, Article ID 465898, 2010.

[44] H. Mahto, R. Tripathy, B. R. Meher et al., “TNF- $\alpha$ promoter polymorphisms (G-238A and G-308A) are associated with susceptibility to systemic lupus erythematosus (SLE) and P. falciparum malaria: a study in malaria endemic area," Scientific Reports, vol. 9, no. 1, p. 11752, 2019.

[45] V. D. Umare, V. D. Pradhan, A. G. Rajadhyaksha, M. M. Patwardhan, K. Ghosh, and A. H. Nadkarni, "Impact of TNF- $\alpha$ and LT $\alpha$ gene polymorphisms on genetic susceptibility in Indian SLE patients," Human Immunology, vol. 78, no. 2, pp. 201-208, 2017.

[46] Y. F. Zou, X. L. Feng, J. H. Tao et al., "Meta-analysis of TNF- $\alpha$ promoter $-308 \mathrm{~A} / \mathrm{G}$ polymorphism and SLE susceptibility in Asian populations," Rheumatology International, vol. 31, no. 8, pp. 1055-1064, 2011.

[47] Y. Tang, H. Tao, Y. Gong, F. Chen, C. Li, and X. Yang, "Changes of serum IL-6, IL-17, and complements in systemic lupus erythematosus patients," Journal of Interferon \& Cytokine Research, vol. 39, no. 7, pp. 410-415, 2019.

[48] N. Ruchakorn, P. Ngamjanyaporn, T. Suangtamai et al., "Performance of cytokine models in predicting SLE activity," Arthritis research \& therapy, vol. 21, no. 1, p. 287, 2019.

[49] A. S. Shaltout, D. Sayed, M. S. Badary et al., "Effect of IL6 and IL23 on double negative T cells and anti ds-DNA in systemic lupus erythematosus patients," Human Immunology, vol. 77, no. 10, pp. 937-943, 2016.

[50] K. Tominaga, T. Yoshimoto, K. Torigoe et al., "IL-12 synergizes with IL-18 or IL-1beta for IFN-gamma production from human T cells," International Immunology, vol. 12, no. 2, pp. 151-160, 2000.

[51] K. B. Onel, D. Huo, D. Hastings, J. Fryer-Biggs, M. K. Crow, and K. Onel, "Lack of association of the TP53 Arg72Pro SNP and the MDM2 SNP309 with systemic lupus erythematosus in Caucasian, African American, and Asian children and adults," Lupus, vol. 18, no. 1, pp. 61-66, 2009.

[52] J. Yang, J. M. Zhu, S. Wu et al., "Association study between the TP53 Rs1042522G/C polymorphism and susceptibility to systemic lupus erythematosus in a Chinese Han population," Rheumatology International, vol. 37, no. 4, pp. 523-529, 2017.

[53] Z. Teng, X. Lin, C. Luan, Y. Sun, and X. Li, “The high expression of miR-564 in patients with systemic lupus erythematosus promotes differentiation and maturation of DC cells by negatively regulating TP53 expression in vitro," Lupus, vol. 30, no. 9, pp. 1469-1480, 2021.

[54] A. N. Beşliu, G. Pistol, C. M. Marica et al., "PI3K/Akt signaling in peripheral T lymphocytes from systemic lupus erythematosus patients," Roumanian Archives of Microbiology and Immunology, vol. 68, no. 2, pp. 69-79, 2009.

[55] D. Iacobas, J. Wen, S. Iacobas, N. Schwartz, and C. Putterman, "Remodeling of neurotransmission, chemokine, and PI3KAKT signaling genomic fabrics in neuropsychiatric systemic lupus erythematosus," Genes (Basel), vol. 12, no. 2, p. 251, 2021.

[56] C. Zhao, Y. Gu, L. Chen, and X. Su, "Upregulation of FoxO3a expression through PI3K/Akt pathway attenuates the progression of lupus nephritis in MRL/lpr mice," International Immunopharmacology, vol. 89, p. 107027, 2020.

[57] G. Guo, Y. Meng, W. Tan et al., "Induction of apoptosis coupled to endoplasmic reticulum stress through regulation 
of CHOP and JNK in bone marrow mesenchymal stem cells from patients with systemic lupus erythematosus," Journal of Immunology Research, vol. 2015, Article ID 183738, 2015.

[58] Y. Molad, M. Amit-Vasina, O. Bloch, E. Yona, and M. J. Rapoport, "Increased ERK and JNK activities correlate with disease activity in patients with systemic lupus erythematosus," Annals of the Rheumatic Diseases, vol. 69, no. 1, pp. 175-180, 2010.

[59] O. Bloch, M. Amit-Vazina, E. Yona, Y. Molad, and M. J. Rapoport, "Increased ERK and JNK activation and decreased ERK/JNK ratio are associated with long-term organ damage in patients with systemic lupus erythematosus," Rheumatology (Oxford, England), vol. 53, no. 6, pp. 1034-1042, 2014. 